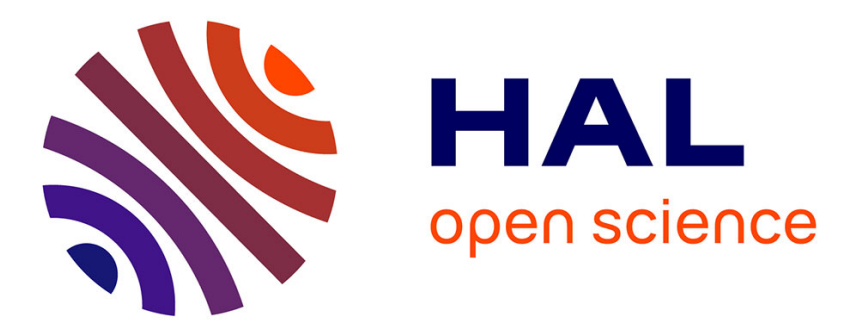

\title{
A general global-local modelling framework for the deterministic optimisation of composite structures
}

Marco Picchi Scardaoni, Marco Montemurro

\section{To cite this version:}

Marco Picchi Scardaoni, Marco Montemurro. A general global-local modelling framework for the deterministic optimisation of composite structures. Structural and Multidisciplinary Optimization, 2020, 10.1007/s00158-020-02586-4 . hal-02914952

\section{HAL Id: hal-02914952 \\ https://hal.inrae.fr/hal-02914952}

Submitted on 22 Sep 2020

HAL is a multi-disciplinary open access archive for the deposit and dissemination of scientific research documents, whether they are published or not. The documents may come from teaching and research institutions in France or abroad, or from public or private research centers.
L'archive ouverte pluridisciplinaire HAL, est destinée au dépôt et à la diffusion de documents scientifiques de niveau recherche, publiés ou non, émanant des établissements d'enseignement et de recherche français ou étrangers, des laboratoires publics ou privés. 


\title{
A General Global-Local Modelling Framework for the Deterministic Optimisation of Composite Structures *
}

\author{
Marco Picchi Scardaoni ${ }^{\dagger 1,2}$ and Marco Montemurro ${ }^{\ddagger 1, *}$ \\ ${ }^{1}$ Arts et Métiers Institute of Technology, Université de Bordeaux, CNRS, INRA, Bordeaux \\ INP, HESAM Université, I2M UMR 5295, F-33405 Talence, France \\ ${ }^{2}$ University of Pisa, Department of Civil and Industrial Engineering, Aerospace Division, Via \\ G. Caruso 8, 56124, Pisa, Italy \\ ${ }^{*}$ Corresponding author
}

May 27, 2020

\begin{abstract}
This work deals with the multi-scale optimisation of composite structures by adopting a general global-local (GL) modelling strategy to assess the structure responses at different scales. The GL modelling approach is integrated into the multi-scale two-level optimisation strategy (MS2LOS) for composite structures. The resulting design strategy is, thus, called GL-MS2LOS and aims at proposing a very general formulation of the design problem, without introducing simplifying hypotheses on the laminate stack and by considering, as design variables, the full set of geometric and mechanical parameters defining the behaviour of the composite structure at each pertinent scale. By employing a GL modelling approach, most of the limitations of well-established design strategies, based on analytical or semi-empirical models, are overcome. The GL-MS2LOS makes use of the polar formalism to describe the anisotropy of the composite at the macroscopic scale (where it is modelled as an equivalent homogeneous anisotropic plate). In this work, deterministic algorithms are exploited during the solution search phase. The challenge, when dealing with such a design problem, is to develop a suitable formulation and dedicated operators, to link global and local models physical responses and their gradients. Closed-form expressions of structural responses gradients are rigorously derived by taking into account for the coupling effects when passing from global to local models. The effectiveness of the GL-MS2LOS is proven on a meaningful benchmark: the least-weight design of a cantilever wing subject to different design requirements. Constraints include maximum allowable displacements, maximum allowable strains, blending, manufacturability requirements and buckling factor.
\end{abstract}

Keywords - Composites, Anisotropy, Polar method, Deterministic Optimisation, Buckling, Global/local modelling approach

\section{Introduction}

Last decades have seen an increasing interest in the study and in the use of composite materials, especially for aeronautical structures. Composites materials allow for a greater freedom in the design process when compared to metal alloys. In fact, the material itself can be tailored according to the requirements of the problem at hand. Furthermore, high specific stiffness and specific strength make composite materials appealing for the aeronautical industry.

Despite these advantages, many difficulties arise since the initial design phases. Issues are mainly related to the mathematical description of anisotropy, as well as to the scale separation between the

\footnotetext{
${ }^{*}$ This is a pre-print of an article published in Structural and Multidisciplinary Optimization. The final authenticated version is available online at: https://doi.org/10.1007/s00158-020-02586-4

${ }^{\dagger}$ marco.picchiscardaoni@ing.unipi.it, marco.picchi_scardaoni@ensam.eu

${ }^{\ddagger}$ marco.montemurro@ensam.eu, marco.montemurro@u-bordeaux.fr
} 
most important physical responses to be integrated into the design process.

As far as the mathematical description of the anisotropy is concerned, the most common multi-scale optimisation approaches available in the literature make use of the well-known lamination parameters (LPs) coupled with the parameters of Tsai and Pagano, see Jones (2018); Tsai and Pagano (1968); Tsai and Hahn (1980). These parameters unquestionably provide a compact representation of the stiffness tensors of the laminate; although, they are not all tensor invariants, as discussed in Tsai and Hahn (1980). Moreover, both LPs and Tsai and Pagano parameters have not an immediate physical meaning related to the elastic symmetries of the laminate stiffness tensor.

A sound alternative for describing the anisotropic behaviour of composite materials and structures is represented by the polar formalism introduced by Verchery (1982). Thanks to the polar formalism it is possible to represent any plane tensor by means of tensor invariants, referred as polar parameters (PPs), which are related to the symmetries of the tensor. In particular, for a fourth-order elasticity-like plane tensor (i.e. a tensor having both major and minor symmetries), all possible elastic symmetries can be easily expressed in terms of conditions on the tensor PPs. Moreover, the polar formalism offers a frame-invariant description of any plane tensor, see Verchery (1982). Moreover, recently, the polar method has been generalised to the case of higher-order equivalent single layer theories in Montemurro $(2015 \mathrm{a}, \mathrm{b}, \mathrm{c})$.

Regarding the intrinsic multi-scale nature of the design problem of composite structures, the most common approach used in the literature is to transform the design problem into a multi-scale twolevel optimisation problem, see for example Albazzan et al. (2019). At the first level of the strategy, each laminate composing the structure is considered as a homogeneous anisotropic equivalent singlelayer plate: the goal is to find the optimal distribution of the geometrical and mechanical (i.e. LPs or PPs depending on the anisotropy description) design variables satisfying the requirements of the problem at hand. The second level of the strategy focuses on the laminate mesoscopic scale and aims at retrieving, at least, one optimum stacking sequence meeting the optimal stiffness properties resulting from the first-level problem.

Some interesting works based on the LPs for describing the macroscopic response of the laminate can be found in Liu et al. (2012); Herencia et al. (2008). Conversely, the multi-scale two-level optimisation strategy (MS2LOS) based on the polar formalism has been originally introduced in Montemurro et al. (2012a,b). The MS2LOS, coupled with polar formalism, has been successfully used in several works, such as Montemurro et al. (2016, 2013); Catapano and Montemurro (2014b,a); Montemurro et al. (2018), and extended to Variable Angle Tow (VAT) composites (Montemurro and Catapano, 2019, 2016, 2017). Furthermore, recently, an experimental validation of the effectiveness of the MS2LOS has been presented in Montemurro et al. (2019).

Due to the discrete variables involved in the definition of a composite laminate (e.g. the number of plies, the orientation angle of each lamina, etc.), the common choice is to use genetic algorithms (GAs) or other metaheuristics to perform the solution search during the optimisation. Nevertheless, an optimisation based on a GA is not suitable for design problems with a large number of variables. Albeit a deterministic approach is more suited, the price to pay is to transform discrete variables into continuous ones (to be rounded at the end of the procedure) and to accept local optimal solution instead of global ones.

Problems with large number of variables are typical of Topology Optimisation (TO), see for instance the works by Bendsøe and Sigmund (2004); Costa et al. (2017, 2019a,b). The common approach is to use deterministic algorithms together with the analytic expressions of gradients of objective function and constraints, in order to reduce the computational cost. Of course, a similar approach can be used for the multi-scale design of composite structures, by paying a particular attention on the strong non-convexity of the resulting optimisation problem. In the last 30 years, some efforts have been done to employ deterministic optimisation methods for the multi-scale design of composites by considering different physical responses, like compliance, buckling, strength, etc. The first attempts dealing with the integration of a requirement on the first buckling factor of the structure can be found in the works by Rodrigues et al. (1995) and by Neves et al. (1995), where authors use the adjoint method to derive, with a variational approach, the analytic expression of the buckling factor gradient. Setoodeh et al. (2009) introduced the decomposition of the geometric stiffness matrix of a single element as a linear 
combination of in-plane forces per unit length and some matrices depending only on the geometry of the element. However, they do not provide the expressions of these matrices, and they do not consider the contributions due to transverse shear stresses. In the article of IJsselmuiden et al. (2009), authors deal with blending and buckling in a gradient-based optimisation framework. However, buckling is formulated via an empirical (approximated) formula. The same authors, in Ijsselmuiden et al. (2010), use the approach of Setoodeh et al. (2009) for VAT buckling load maximisation. Interesting is the work of Bian and Fang (2017), who propose an assembly-free method for TO. In Thomsen et al. (2018), authors derive the buckling gradient for TO problems in the framework of the Solid Isotropic Material Penalisation (SIMP) approach. Ferrari and Sigmund (2019) revisit the buckling constraint for TO and study the influence of common practices, such as inconsistent gradients, obtained by neglecting some terms as done in the works by Ye et al. (2015); Munk et al. (2016). Finally, Townsend and Kim (2019) propose a level-set topology optimisation for buckling of shell-like structures.

Surprisingly, in the aforementioned works, authors do not to include out-of-plane shear stresses contribution in the assembly of the geometric stiffness matrix.

A second major issue in composite laminates design, which is still an open problem, is the so-called blending requirement, initially introduced in Kristinsdottir et al. (2001). The blending requirement consists of ensuring the continuity of ply orientations between adjacent panels having a different number of layers. In the framework of the polar method and the First-order Shear Deformation Theory (FSDT) of laminates, the recent work by Panettieri et al. (2019) proposes a general approach consisting in a set of four inequalities on the laminate PPs, to be imposed in the first-level of the MS2LOS, in order to ensure the recovery of blended stacking sequences in the second-level problem. For an exhaustive literature survey on blending, the reader is addressed to Panettieri et al. (2019).

A further issue in the design of composite structures is related to the mathematical model, adopted at each pertinent scale, to assess the physical responses involved in the design problem. In order to reduce the computational cost of the whole optimisation process, in all the studies mentioned beforehand, analytical (approximate) models are used for the assessment of the response of the structure. Accordingly, the main limitations of these approaches are the lack of accuracy and the limited applicability of such methods that rely on simplifying hypotheses, especially in terms of applied boundary conditions (BCs), which are non-representative of real operative conditions.

To go beyond these limitations, some authors proposed the use of enhanced semi-analytical formulations to assess local structural responses. For example in Bisagni and Vescovini (2015), the authors proposed an analytical formulation, based on the Rayleigh-Ritz method, able to better describe the interaction between the skin and the stringers due to buckling, but still neglecting the frames compliance and considering the structure infinitely periodic. In other works, like Irisarri et al. (2011); Vankan et al. (2014), surrogate models built from results of FE analyses are employed, but the problem of the representativeness of the BCs still persist and the phenomenon of mode switching can lead to a further inaccuracy in the evaluation of the buckling response. Therefore, the use of a proper finite element (FE) modelling strategy, for both global and local scales phenomena assessment, should be used in these situations. However, as pointed out by Venkataraman and Haftka (2004), its integration in a multi-scale optimisation strategy could be difficult when complex structures characterised by many design variables are considered.

To overcome the aforementioned issues, a dedicated global-local (GL) FE modelling approach, initially presented in Izzi et al. (2020) for optimisation of composite structures based on GAs, is here improved for deterministic algorithms and integrated in the MS2LOS of composite structures. The resulting methodology is referred as GL-MS2LOS. GL modelling strategies are quite commonly used in the structural analysis of the aeronautical structures (Izzi et al., 2020; Ciampa et al., 2010; Liu et al., 2016). However, GL modelling strategies are rarely coupled to deterministic optimisation methods due to the following issues:

1. the high computational costs;

2. the lack of pertinent criteria to identify the zones of interest (ZOIs) within the global FE model (GFEM);

3. the lack of suitable modelling strategies to automatically build the local FE models (LFEMs) 
by extracting pertinent information from the GFEM and by taking into account for variable (possibly discontinuous) geometry and mesh;

4. the lack of a proper mathematical formulation to derive the gradient of the physical responses resulting from LFEMs with respect to the design variables describing the model at each pertinent scale (and the related coupling effect between GFEM and LFEMs responses and their derivatives).

In this scenario, the major contribution of this work is twofold. On the one hand, a closed-form derivation of a large variety of constraints (and their gradients with respect to the design variables), including feasibility, strength, stiffness, blending and buckling factor in the framework of the FSDT is presented, and their expression is derived in the PPs space. On the other hand, a proper GL modelling strategy, based on the sub-modelling technique by Sun and Mao (1988); Mao and Sun (1991); Whitcomb (1991), has been embedded in the optimisation work-flow and considered in the derivation of such constraints, in particular for the analytic expression of the buckling factor (by taking into account for the coupling effects between LFEM and GFEM).

The paper is organised as follows: a general overview on the design problem and of the MS2LOS is presented in Section 2. The mathematical framework of the first-level problem formulation is detailed in Section 3. A concise description of the FE models, at both global and local levels, are given in Section 4, while numerical results are shown and commented in Section 5. Finally, Section 6 is dedicated to concluding remarks, whilst Appendices A, B, C and D are dedicated to the mathematical proofs for deriving the expression of the gradient of the considered physical and geometrical responses.

Notation: Upper-case bold letters are used to indicate tensors (matrices), while lower-case bold letters indicate vectors, which are to be intended as column ones.

The writing $\operatorname{diag}(a, b, c)$ (possibly $\operatorname{diag}(\mathbf{A}, \mathbf{B}, \mathbf{C})$ ) denotes the diagonal matrix with elements a, b, c (possibly matrices $\mathbf{A}, \mathbf{B}, \mathbf{C}$ ) on the principal diagonal (possibly principal diagonal blocks). $\mathbb{R}^{+}:=\{x \mid$ $x \in \mathbb{R}, x>0\}$ denotes the set of all strictly positive real numbers. $\mathbb{M}^{\mathrm{m} \times \mathrm{n}}$ denotes the set of all realvalued $\mathrm{m} \times \mathrm{n}$ matrices, $\mathbb{M}_{+}^{\mathrm{n} \times \mathrm{n}}:=\left\{\mathbf{M} \mid \mathbf{M} \in \mathbb{M}^{\mathrm{n} \times \mathrm{n}}\right.$, det $\left.\mathbf{M}>0\right\}, \mathbb{M}_{s}^{\mathrm{n} \times \mathrm{n}}:=\left\{\mathbf{M} \mid \mathbf{M} \in \mathbb{M}^{\mathrm{n} \times \mathrm{n}}, \mathbf{M}=\mathbf{M}^{\mathrm{T}}\right\}$, $\mathbb{M}_{s+}^{\mathrm{n} \times \mathrm{n}}:=\mathbb{M}_{+}^{\mathrm{n} \times \mathrm{n}} \cap \mathbb{M}_{s}^{\mathrm{n} \times \mathrm{n}}$ and $\mathbb{M}_{s++}^{\mathrm{n} \times \mathrm{n}}:=\left\{\mathbf{M} \mid \mathbf{M} \in \mathbb{M}_{s+}^{\mathrm{n} \times \mathrm{n}}\right.$, with $\mathbf{M}$ positive - definite $\}$. Finally, $\sharp \mathbf{v}$ denotes the cardinality of the generic vector $\mathbf{v}$, superscript $\hat{\bullet}$ denotes the non-reduced $\bullet$, and label $b$ denotes quantities evaluated in the LFEM.

\section{Multi-Scale Optimisation of Composite Laminates}

\subsection{Problem Description: Simplified Wing-Box Structure}

In order to prove the effectiveness of the GL-MS2LOS, a numerical benchmark, taken from Panettieri et al. (2019); Liu et al. (2011, 2000), has been considered. The benchmark consists in a simplified wing-box model, made of composite laminates, subject to bending and twist moment at the tip section, with a clamped root section. The geometry of the structure is presented in Figure 1. The wing-box has length $\mathrm{L}=3543 \mathrm{~mm}$, width $\mathrm{W}=2240 \mathrm{~mm}$ and height $\mathrm{H}=381 \mathrm{~mm}$. Loads and BCs are deeply discussed in Section 4. In the model, ribs, spars and stringers are replaced by continuous equally spaced composite plates with a pre-defined stacking sequence: $\left[\left( \pm 45^{\circ}\right)_{11}\right]_{S}$. The constitutive ply, used for all laminates composing the structure, is made of a carbon-epoxy T300/5208 pre-preg (Zhang et al., 2019; Catapano, 2013), whose properties are listed in Table1, in terms of both technical constants and PPs. Optimisation regions are dorsal and ventral skins of the wing-box. Each skin is subdivided into nine regions (or panels), numbered as in Fig. 2, for a total number of panels $n_{\text {pan }}$ equal to 18 .

\subsection{Multi-Scale Two-Level Strategy}

Generally speaking, the main goal of the MS2LOS is the minimisation of a merit function (in this case, the mass of the simplified wing-box), subject to requirements of different nature, e.g. geometrical, 


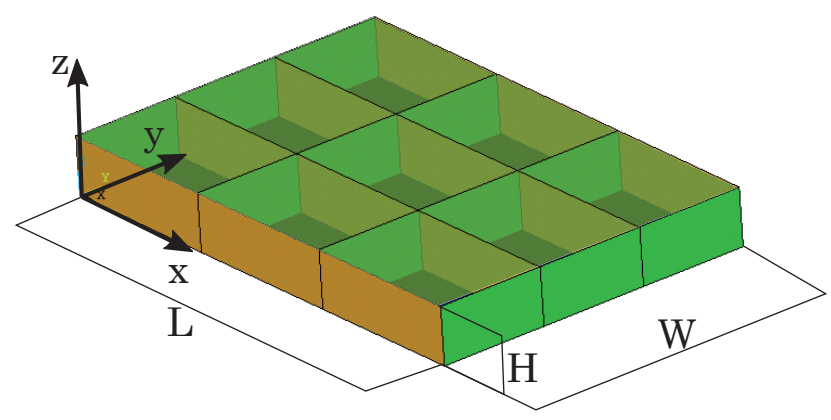

Figure 1: Model geometry

Table 1: Material properties of the T300/5208 pre-preg

\begin{tabular}{|c|c|c|c|c|c|}
\hline \multicolumn{2}{|c|}{ Technical constants } & \multicolumn{2}{|c|}{ Polars parameters of $\mathbf{Q}^{a}$} & \multicolumn{2}{|c|}{ Polars parameters of $\hat{\mathbf{Q}}^{b}$} \\
\hline$E_{1}[\mathrm{GPa}]$ & 181 & $T_{0}[\mathrm{MPa}]$ & 26898.9668 & $T[\mathrm{MPa}]$ & 5398.3802 \\
\hline$E_{2}[\mathrm{GPa}]$ & 10.3 & $T_{1}[\mathrm{MPa}]$ & 24710.2593 & $R[\mathrm{MPa}]$ & 1771.6197 \\
\hline$G_{12}[\mathrm{GPa}]$ & 7.17 & $R_{0}[\mathrm{MPa}]$ & 19728.9668 & $\Phi[\mathrm{deg}]$ & 90 \\
\hline$G_{23}[\mathrm{GPa}]$ & 3.78 & $R_{1}[\mathrm{MPa}]$ & 21426.3863 & & \\
\hline$G_{13}[\mathrm{GPa}]$ & 7.17 & $\Phi_{0}[\mathrm{deg}]$ & 0 & & \\
\hline$\nu_{12}$ & 0.27 & $\Phi_{1}[\mathrm{deg}]$ & 0 & & \\
\hline$\nu_{23}$ & 0.42 & & & & \\
\hline$\nu_{13}$ & 0.27 & & & & \\
\hline \multicolumn{2}{|c|}{ Density and thickness } & \multicolumn{2}{|c|}{ Polars parameters of $\mathbf{G}^{c}$} & \multicolumn{2}{|c|}{ Polars parameters of $\hat{\mathbf{G}}^{d}$} \\
\hline$\rho_{\text {ply }}\left[\mathrm{kg} \mathrm{mm}^{-3}\right]$ & $1.76 \times 10^{-6}$ & $\Gamma_{0}$ & 7531.0292 & $\Gamma$ & 10633.5320 \\
\hline$t_{\mathrm{ply}}[\mathrm{mm}]$ & 0.125 & $\Gamma_{1}$ & 2113.8030 & $\Lambda$ & 484.3096 \\
\hline$n_{\text {ref }}$ & 150 & $\Lambda_{0}$ & 3586.8124 & $\Omega[\mathrm{deg}]$ & 90 \\
\hline & & $\Lambda_{1}$ & 1603.3669 & & \\
\hline & & $\Omega_{0}[\mathrm{deg}]$ & 45 & & \\
\hline & & $\Omega_{1}[\mathrm{deg}]$ & 0 & & \\
\hline \multicolumn{6}{|c|}{ Limit stresses (for Tsai-Hill criterion) } \\
\hline$X[\mathrm{MPa}]$ & 1500 & & & & \\
\hline$Y[\mathrm{MPa}]$ & 246 & & & & \\
\hline$S_{12}[\mathrm{MPa}]$ & 68 & & & & \\
\hline$S_{23}[\mathrm{MPa}]$ & 36 & & & & \\
\hline$S_{13}[\mathrm{MPa}]$ & 68 & & & & \\
\hline
\end{tabular}

\footnotetext{
${ }^{a}$ In-plane ply stiffness matrix

${ }^{b}$ Out-of-plane ply shear stiffness matrix

${ }^{c}$ In-plane ply strength matrix

${ }^{d}$ Out-of-plane ply shear strength matrix
}

technological and physical responses. The global optimisation problem can be split into two distinct (but related) optimisation problems.

1. First-level problem. The aim of this phase, which focuses on the laminate macroscopic scale, is the determination of the optimum distribution of the mechanical and geometric design variables, describing the behaviour of each laminate, in order to minimise the considered objective function and to satisfy the set of design requirements (formulated as optimisation constraints). At this level, the generic laminate is modelled as an equivalent homogeneous orthotropic plate, whose anisotropy is described in terms of laminate PPs (see Vannucci (2017) for a primer). Thanks to this formulation, at this stage the designer can add further requirements (e.g. manufacturability constraints, strength and damage criteria, etc.) by introducing suitable constraints on the laminate PPs. 


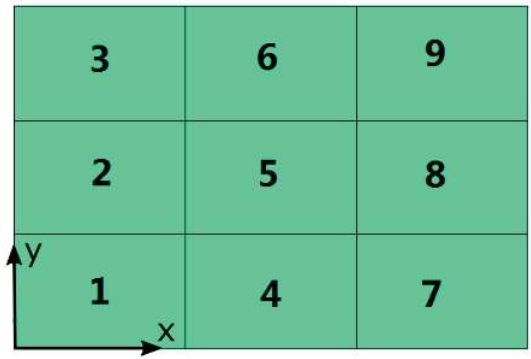

(a) Dorsal laminates numbering

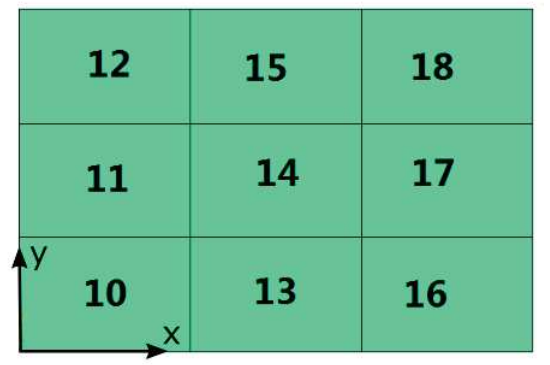

(b) Ventral laminates numbering

Figure 2: Optimisation regions

2. Second-level problem. The second level of the strategy focuses on the laminate mesoscopic scale, and aims at determining optimal stacking sequences, possibly blended between adjacent laminates, in such a way to recover the optimised stiffness properties resulting from the first-level problem. The design variables of the second-level problem are the plies orientation angles.

In this work, only the first-level problem is considered. Further details about the formulation of the second-level problem, and the related numerical strategy, can be found in Montemurro et al. (2019, 2012a,b).

\section{Mathematical Formulation of the First-Level Problem}

The aim of the first-level problem is the mass minimisation of the simplified wing-box structure, represented in Fig. 1, subject to the following design requirements:

- feasibility constraints on the laminate PPs, presented in Vannucci (2012);

- blending constraints among each pair of adjacent panels, expressed as equivalent constraints on the laminate PPs and thickness, as discussed in Panettieri et al. (2019);

- a constraint on the maximum displacement measured on the GFEM;

- a constraint on the laminate maximum failure index evaluated on the check zone in the GFEM;

- a constraint on the first buckling factor of each dorsal sub-panel, evaluated by means of the GL modelling approach on each LFEM;

\subsection{Mechanical and Geometric Design Variables}

The constitutive law of a multilayer plate, expressed in the global frame of the laminate, reads

$$
\mathbf{r}=\mathbf{K}_{\mathrm{lam}} \varepsilon_{g e n}
$$

where $\mathbf{r}$ and $\varepsilon_{\text {gen }}$ are the vectors of the generalised forces per unit length and generalised strains of the laminate middle plane, respectively, whilst $\mathbf{K}_{\text {lam }} \in \mathbb{M}_{s++}^{8 \times 8}$ is the stiffness matrix of the laminate (Voigt's notation).

In the framework of the FSDT (Reddy (2003)), the analytic expressions of these arrays are

$$
\mathbf{r}=\left\{\begin{array}{c}
\mathbf{n} \\
\mathbf{m} \\
\mathbf{t}
\end{array}\right\}, \mathbf{K}_{\mathrm{lam}}=\left[\begin{array}{ccc}
\mathbf{A} & \mathbf{B} & \mathbf{O} \\
& \mathbf{D} & \mathbf{O} \\
\mathrm{sym} & & \mathbf{H}
\end{array}\right], \boldsymbol{\varepsilon}_{\mathrm{gen}}=\left\{\begin{array}{c}
\varepsilon_{0} \\
\boldsymbol{\chi}_{0} \\
\gamma_{0}
\end{array}\right\}
$$

In Eq. (2), $\mathbf{A} \in \mathbb{M}_{s++}^{3 \times 3}$ is the membrane stiffness matrix, $\mathbf{D} \in \mathbb{M}_{s++}^{3 \times 3}$ is the bending stiffness matrix, $\mathbf{H} \in \mathbb{M}_{s++}^{2 \times 2}$ is the out-of-plane shear stiffness matrix, $\mathbf{B} \in \mathbb{M}_{s}^{3 \times 3}$ is the membrane/bending coupling stiffness matrix. Vectors $\mathbf{n}, \mathbf{m}, \mathbf{t}$ are the membrane forces, bending moments and out-of-plane shear 
forces per unit length, respectively. Vectors $\varepsilon_{0}, \chi_{0}, \gamma_{0}$ represent the in-plane strains, curvatures and out-of-plane shear strains of the laminate middle plane, respectively.

It is convenient to introduce the following homogenised tensors:

$$
\mathbf{A}^{*}:=\frac{1}{h} \mathbf{A}, \quad \mathbf{B}^{*}:=\frac{2}{h^{2}} \mathbf{B}, \quad \mathbf{D}^{*}:=\frac{12}{h^{3}} \mathbf{D}, \quad \mathbf{H}^{*}:=\frac{1}{h} \mathbf{H},
$$

where $h$ is the total thickness of the laminate.

As shown in Montemurro (2015a,b), in the framework of the polar formalism, in the case of fully orthotropic, quasi-homogeneous laminates, the overall number of independent variables does not change if FSDT is considered, instead of the classic laminate theory (CLT). In particular, once the basic ply is chosen, only three independent variables are needed to completely characterise the laminate behaviour, namely $R_{0 K}^{A^{*}}, R_{1}^{A^{*}}, \Phi_{1}^{A^{*}}$. The fourth variable needed to describe the laminate response is the number of plies $n_{\text {ply }}$, which is defined as $n_{\text {ply }}=h / t_{\text {ply }}$, where $t_{\text {ply }}$ is the thickness of the single ply. For more details on the polar formalism, the reader is addressed to Verchery (1982); Vannucci (2017, 2005); Montemurro (2015a,b).

For optimisation purposes, it is useful to introduce the dimensionless PPs of $\mathbf{A}^{*}$ and the dimensionless number of plies, as follows:

$$
n_{0}:=\frac{n_{\mathrm{ply}}}{n_{\mathrm{ref}}}, \quad \rho_{0 K}:=\frac{R_{0 K}^{A^{*}}}{R_{0}}, \quad \rho_{1}:=\frac{R_{1}^{A^{*}}}{R_{1}}, \quad \phi_{1}:=\frac{\Phi_{1}^{A^{*}}}{\pi / 2},
$$

where $n_{\text {ref }}$ is the reference value of the number of plies, whilst $R_{0}$ and $R_{1}$ are the anisotropic moduli of the constitutive lamina; the value of these quantities is reported in Table 1 . The expression of the laminate homogenised stiffness matrices of Eq. (3) in terms of the laminate dimensionless design variables of Eq. (4), together with their gradient, is given in Appendix A.

Hence, for each one of the $n_{\text {pan }}$ laminates composing the wing-box, i.e. for each region where (4) assume constant values, four variables are needed. Design variables can be collected in the vector:

$$
\xi^{\mathrm{T}}:=\left\{n_{0}^{i}, \rho_{0 K}^{i}, \rho_{1}^{i}, \phi_{1}^{i} \mid i=1, \cdots, n_{\mathrm{pan}}\right\} .
$$

The total number of design variables is then equal to $n_{\mathrm{vars}}:=\sharp \boldsymbol{\xi}=4 n_{\mathrm{pan}}$.

\subsection{Laminate Feasibility Constraints}

In the first level of the MS2L strategy, geometric and feasibility constraints on the PPs must be considered (Vannucci, 2012), in order to ensure that the optimal values of PPs correspond to a feasible stacking sequence (to be determined during the second step of the procedure). Since orthotropy and quasi-homogeneity (i.e. uncoupling with the same group of symmetry in membrane and bending) properties holds, such constraints read (Vannucci, 2012):

$$
\left\{\begin{array}{l}
-1 \leq \rho_{0 K} \leq+1 \\
0 \leq \rho_{1} \leq 1 \\
2 \rho_{1}^{2}-1-\rho_{0 K} \leq 0
\end{array}\right.
$$

The first two equations of (6) can be considered as bounds for the design variables; therefore, the feasibility constraint is represented only by the third equation of (6), which must be evaluated $n_{\text {pan }}$ times, i.e. for each panel of the wing-box.

However, in order to avoid a large number of constraints (and gradients), which may lead to a over-constrained optimisation problem, one may use suitable aggregation strategies, e.g. by introducing the well-know maximum operator. Inasmuch as max operator is not everywhere differentiable with continuity $\left(\mathcal{C}^{0}\right.$ class), a continuous and differentiable approximation (at least of class $\left.\mathcal{C}^{1}\right)$ must be introduced.

Let $\mathbf{x}$ be a generic vector, whose cardinality is $n$, and let introduce the following operator, called 
$\alpha$-LogSumExp, often used in machine learning and artificial neural networks (Haykin, 1998; Nielsen and Sun, 2016; Calafiore and El Ghaoui, 2014):

$$
\operatorname{LSE}_{\alpha}(\mathbf{x}):=\frac{1}{\alpha} \ln \left(\sum_{i=1}^{n} e^{\alpha x_{i}}\right),
$$

where $\alpha$ is a parameter which can be chosen according to the variance $\operatorname{Var}(\mathbf{x})$ and to the values of vector $\mathbf{x}$. The more $\alpha>>1$, the better $\operatorname{LSE}_{\alpha} \rightarrow \max \mathbf{x}$.

It can be easily shown that the family LSE $\mathrm{L}_{\alpha}$ approaches the max by excess; in particular, the following estimate holds:

$$
\max \mathbf{x} \leq \mathrm{LSE}_{\alpha} \leq \max \mathbf{x}+\frac{1}{\alpha} \ln (n) .
$$

Equation (8) is much more accurate as $\operatorname{Var}(\mathbf{x})$ and/or $\alpha$ assume large values. Furthermore, LSE $\mathrm{L}_{\alpha}$ is a convex and strictly-monotonically-increasing function in its domain (Calafiore and El Ghaoui, 2014). Relation (8) is a fundamental inequality, since it ensures that the approximated constraints are conservative. The approximation consists in considering a value of $\alpha$ reasonably large, in order to avoid too sharp approximations and numerical overflows on the one hand, but maintaining the discrete effect of the max operator and to reasonably approximate the maximum value on the other hand.

In this case, the $n_{\text {pan }}$ inequalities of (6), collected in the vector $\mathbf{g}$, can be replaced by the condition

$$
g_{\text {feas }}:=\operatorname{LSE}_{\alpha}(\mathbf{g}), \quad \alpha>>1 .
$$

The gradient of of Eq. (9), with respect to the generic design variable $\xi_{j}$, reads:

$$
\left(\nabla_{\boldsymbol{\xi}} g_{\mathrm{feas}}\right)_{j}:=\frac{\partial g_{\mathrm{feas}}}{\partial \xi_{j}}=\frac{\sum_{i=1}^{n_{\mathrm{pan}}} e^{\alpha g_{i}} \frac{\partial g_{i}}{\partial \xi_{j}}}{\sum_{i=1}^{n_{\mathrm{pan}}} e^{\alpha g_{i}}}, \quad \alpha>>1, \quad j=1, \ldots, n_{\mathrm{vars}}
$$

where

$$
\frac{\partial g_{i}}{\partial \xi_{j}}=\left\{\begin{array}{l}
-1 \text { if } \xi_{j}=\rho_{0 K}^{i} \\
4 \rho_{1}^{i} \text { if } \xi_{j}=\rho_{1}^{i} \\
0 \quad \text { otherwise }
\end{array}\right.
$$

Regarding the parameter $\alpha$, after some empirical tuning, it has been set as follows:

$$
\alpha(\operatorname{Var}(\mathbf{g}))=\left\{\begin{array}{l}
70 \text { if } \operatorname{Var}(\mathbf{g}) \leq 0.001 \\
50 \text { if } 0.001<\operatorname{Var}(\mathbf{g}) \leq 0.1, \\
10 \text { if } \operatorname{Var}(\mathbf{g}) \geq 0.1
\end{array}\right.
$$

\subsection{Displacement Requirement}

The displacement requirement is measured on the GFEM and is considered as the vertical displacement (i.e. along the $z$ axis) of node $\mathrm{A}$ at the tip of the wing-box, as illustrated in Fig. 4.

Let $n_{\mathrm{DOF}}$ denote the number of DOFs of the unconstrained GFEM. Since BCs are applied at $n_{\mathrm{BC}}$ DOFs, the constrained GFEM has $n_{\mathrm{IN}}:=n_{\mathrm{DOF}}-n_{\mathrm{BC}}$ DOFs. Let $\mathbf{u}$ be the solution of the problem:

$$
\mathbf{K u}-\mathbf{f}=\mathbf{0},
$$

where $\mathbf{u}$ is the vector of unknowns nodal displacements and rotations, $\mathbf{f}$ is the vector of external generalised nodal forces, and $\mathbf{K} \in \mathbb{M}_{s++}^{\mathrm{n}_{I N} \times \mathrm{n}_{I N}}$ is the (constrained) stiffness matrix of the GFEM.

Let index $k$ denote the position, in $\mathbf{u}$, of the displacement of node $\mathrm{A}$ along the $z$ axis. It is convenient to introduce the vector a, whose its unique non-zero component has unit value in position $k$, such that

$$
\frac{\partial u_{k}}{\partial \xi_{j}}=\mathbf{a}^{\mathrm{T}} \frac{\partial \mathbf{u}}{\partial \xi_{j}} .
$$


Under the hypothesis that external generalised nodal forces do not depend on design variables:

$$
\frac{\partial \mathbf{f}}{\partial \xi_{j}}=\mathbf{0}, \quad \forall j=1, \ldots, n_{\mathrm{vars}},
$$

Eq.(14) can be augmented with the null quantity obtained by differentiation of Eq.(13):

$$
\frac{\partial u_{k}}{\partial \xi_{j}}=\mathbf{a}^{\mathrm{T}} \frac{\partial \mathbf{u}}{\partial \xi_{j}}+\boldsymbol{\mu}^{\mathrm{T}}\left(\frac{\partial \mathbf{K}}{\partial \xi_{j}} \mathbf{u}+\mathbf{K} \frac{\partial \mathbf{u}}{\partial \xi_{j}}\right) .
$$

The expression of $\partial \mathbf{K} / \partial \xi_{j}$ is given in Appendix B.

The adjoint vector $\boldsymbol{\mu}$ is chosen in such a way that the term multiplying $\frac{\partial \mathbf{u}}{\partial \xi_{j}}$ vanishes from Eq. (16). Finally, if $u_{0}$ denotes the maximum allowable displacement, one obtains

$$
g_{\text {disp }}:=\frac{u_{k}}{u_{0}}-1 \leq 0,
$$

and

$$
\left\{\begin{array}{l}
\left(\nabla_{\boldsymbol{\xi}} g_{\text {disp }}\right)_{j}:=\frac{1}{u_{0}} \frac{\partial u_{k}}{\partial \xi_{j}}=\boldsymbol{\mu}^{\mathrm{T}} \frac{\partial \mathbf{K}}{\partial \xi_{j}} \frac{\mathbf{u}}{u_{0}}, \quad \forall j=1, \ldots, n_{\mathrm{vars}}, \\
\mathbf{K} \boldsymbol{\mu}=-\mathbf{a} .
\end{array}\right.
$$

\subsection{Laminate Failure Criterion}

The requirement on the laminate strength is based on the results of the works made by Catapano et al. (2012, 2014); Catapano and Montemurro (2018). The authors introduce a laminate failure index, in the framework of the FSDT, averaged on the laminate thickness $h$, having the form:

$$
F=\frac{1}{h}\left(\varepsilon_{0}^{\mathrm{T}} \mathbf{G}_{A} \boldsymbol{\varepsilon}_{0}+\boldsymbol{\chi}_{0}^{\mathrm{T}} \mathbf{G}_{D} \boldsymbol{\chi}_{0}+2 \boldsymbol{\varepsilon}_{0}^{\mathrm{T}} \mathbf{G}_{B} \boldsymbol{\chi}_{0}+\boldsymbol{\gamma}_{0}^{\mathrm{T}} \mathbf{G}_{H} \boldsymbol{\gamma}_{0}+\boldsymbol{\varepsilon}_{0}^{\mathrm{T}} \mathbf{g}_{A}+\boldsymbol{\chi}_{0}^{\mathrm{T}} \mathbf{g}_{D}\right)-1 \leq 0,
$$

where $\mathbf{G}_{A}, \mathbf{G}_{B}, \mathbf{G}_{D}, \mathbf{G}_{H}$ are the laminate membrane, membrane/bending coupling, bending and outof-plane shear strength matrices, respectively, while $\mathbf{g}_{A}$ and $\mathbf{g}_{D}$ are the membrane and bending strength vectors related to the linear part of the failure criterion. As discussed in Catapano and Montemurro (2018), laminate stiffness and strength matrices are strictly related: all of the aforementioned quantities can be expressed in terms of the PPs of matrix $\mathbf{A}^{*}$; hence, the number of variables is not increased by the introduction of the laminate strength matrices and vectors (see Appendix $\mathrm{C}$ for the related expressions and gradients).

Under the hypothesis of quasi-homogeneity and by considering the Tsai-Hoffman criterion, Eq. (19) reduces to

$$
F_{\mathrm{TH}}:=\frac{1}{h}\left(\varepsilon_{\text {gen }}^{\mathrm{T}} \mathbf{G} \varepsilon_{\text {gen }}\right)-1 \leq 0, \quad \text { with } \mathbf{G}:=\operatorname{diag}\left(\mathbf{G}_{A}, \mathbf{G}_{D}, \mathbf{G}_{H}\right) .
$$

Eq. (20) is evaluated for all elements of the GFEM belonging to the check zone, where the strain field assumes meaningful values. In this work, the check zone is composed by the elements (whose number is $n_{e}$ ) belonging to the ventral skin on the wing-box of Fig. 1 and $2 \mathrm{~b}$. To avoid a large number of constraints (and gradients), the aggregation based on the maximum operator is used also in this case:

$$
g_{\mathrm{TH}}=\max _{e}\left(\frac{1}{h_{e}} \varepsilon_{\text {gen } e}^{\mathrm{T}} \mathbf{G}_{e} \boldsymbol{\varepsilon}_{\mathrm{gen} e}\right)-1 \leq 0, \quad e=1, \ldots, n_{e} .
$$

Adopting the approximation of Eq. (7), and adding the null quantity of Eq. (13), Eq. (21) becomes:

$$
g_{\mathrm{TH}}=\operatorname{LSE}_{\alpha}(\boldsymbol{\delta})+\boldsymbol{\mu}^{\mathrm{T}}(\mathbf{K u}-\mathbf{f})-1 \leq 0, \quad e=1, \ldots, n_{e}, \quad \alpha>>1,
$$


where vector $\boldsymbol{\delta}$ collects the quantity

$$
\delta_{e}:=\frac{1}{h_{e}} \varepsilon_{\mathrm{gen} e}^{\mathrm{T}} \mathbf{G}_{e} \boldsymbol{\varepsilon}_{\mathrm{gen} e}, \quad e=1, \ldots, n_{e} .
$$

By deriving Eq. (22), remembering assumption (15), one obtains:

$$
\left(\nabla_{\boldsymbol{\xi}} g_{\mathrm{TH}}\right)_{j}:=\frac{\partial g_{\mathrm{TH}}}{\partial \xi_{j}}=\frac{\sum_{e=1}^{n_{e}} e^{\alpha \delta_{e}} \frac{\partial \delta_{e}}{\partial \xi_{j}}}{\sum_{e=1}^{n_{e}} e^{\alpha \delta_{e}}}+\boldsymbol{\mu}^{\mathrm{T}}\left(\frac{\partial \mathbf{K}}{\partial \xi_{j}} \mathbf{u}+\mathbf{K} \frac{\partial \mathbf{u}}{\partial \xi_{j}}\right), \quad \alpha>>1, \quad j=1, \ldots, n_{\mathrm{vars}}
$$

It is convenient to introduce the connectivity matrix of element $e$ (24 are the DOFs of a shell element with four nodes and six DOFs per node) defined as

$$
\mathbf{L}_{e}: \mathbf{u} \mapsto \mathbf{u}_{e}, \quad \mathbf{L}_{e} \mathbf{u}=\mathbf{u}_{e}, \quad \mathbf{L}_{e} \in \mathbb{M}^{24 \times \mathrm{n}_{\mathrm{IN}}}
$$

and the matrix $\mathbf{B}_{e}$, representing the product of the linear differential operator and the shape function matrices, i.e.

$$
\mathbf{B}_{e}: \mathbf{u}_{e} \mapsto \varepsilon_{\text {gen } e}, \quad \mathbf{B}_{e} \mathbf{u}_{e}=\varepsilon_{\text {gen } e}, \quad \mathbf{B}_{e} \in \mathbb{M}^{8 \times 24} .
$$

By injecting Eqs. (25) and (26) in Eq. (23) and by deriving, one obtains:

$$
\begin{aligned}
\frac{\partial \delta_{e}}{\partial \xi_{j}} & :=\boldsymbol{\beta}_{e} \frac{\partial \mathbf{u}}{\partial \xi_{j}}+\eta_{e j}, \text { with : } \\
\boldsymbol{\beta}_{e} & :=\frac{2}{h_{e}} \boldsymbol{\varepsilon}_{\mathrm{gen} e}^{\mathrm{T}} \mathbf{G}_{e} \mathbf{B}_{e} \mathbf{L}_{e}, \eta_{e j}:=\frac{1}{h_{e}}\left(\varepsilon_{\text {gen } e}^{\mathrm{T}} \frac{\partial \mathbf{G}_{e}}{\partial \xi_{j}} \boldsymbol{\varepsilon}_{\mathrm{gen} e}-\delta_{e} \frac{\partial h_{e}}{\partial \xi_{j}}\right) .
\end{aligned}
$$

The expression of $\partial \mathbf{G}_{e} / \partial \xi_{e}$, appearing in the above formula, is provided in Appendix C. By injecting Eq. (27) into Equation (24), one obtains:

$$
\begin{aligned}
& \frac{\partial g_{\mathrm{TH}}}{\partial \xi_{j}}=\eta_{j}+\boldsymbol{\mu}^{\mathrm{T}} \frac{\partial \mathbf{K}}{\partial \xi_{j}} \mathbf{u}+\left(\boldsymbol{\beta}^{\mathrm{T}}+\boldsymbol{\mu}^{\mathrm{T}} \mathbf{K}\right) \frac{\partial \mathbf{u}}{\partial \xi_{j}}, \text { with }: \\
& \boldsymbol{\beta}^{\mathrm{T}}:=\frac{\sum_{e=1}^{n_{e}} \mathrm{e}^{\alpha \delta_{e}} \boldsymbol{\beta}_{e}^{\mathrm{T}}}{\sum_{e=1}^{n_{e}} \mathrm{e}^{\alpha \delta_{e}}}, \eta_{j}:=\frac{\sum_{e=1}^{n_{e}} \mathrm{e}^{\alpha \delta_{e}} \eta_{e j}}{\sum_{e=1}^{n_{e}} \mathrm{e}^{\alpha \delta_{e}}} .
\end{aligned}
$$

In Eq. (28), the vector $\boldsymbol{\mu}$ can be chosen in such a way that the term multiplying $\frac{\partial \mathbf{u}}{\partial \xi_{j}}$ vanishes; accordingly, one finally obtains the following expression:

$$
\left\{\begin{array}{l}
\left(\nabla_{\boldsymbol{\xi}} g_{\mathrm{TH}}\right)_{j}:=\eta_{j}+\boldsymbol{\mu}^{\mathrm{T}} \frac{\partial \mathbf{K}}{\partial \xi_{j}} \mathbf{u}, \quad j=1, \ldots, n_{\mathrm{vars}} \\
\mathbf{K} \boldsymbol{\mu}=-\boldsymbol{\beta} \\
\alpha>>1
\end{array}\right.
$$

After some empirical tuning, the parameter $\alpha$ has been set as follows:

$$
\alpha(\operatorname{Var}(\boldsymbol{\delta}))= \begin{cases}50 & \text { if } \operatorname{Var}(\boldsymbol{\delta}) \leq 0.001 \\ 30 & \text { if } 0.001<\operatorname{Var}(\boldsymbol{\delta}) \leq 0.1 \\ 10 & \text { if } 0.1<\operatorname{Var}(\boldsymbol{\delta}) \leq 0.3 \\ 2 & \text { if } \operatorname{Var}(\boldsymbol{\delta}) \geq 0.3\end{cases}
$$




\subsection{Blending Requirement}

The formulation of blending constraints in the PPs space used in this study is taken from Panettieri et al. (2019). Blending represents a major issue in composite structures: the continuity of ply orientations between adjacent laminates must be addressed since the first design stages, in order to find optimal solutions which are also realisable. If indices $p$ and $q$ denote a generic couple of adjacent laminates, in the PPs space, blending constraints read:

$$
g_{\text {blend }}^{p q}=\left\{\begin{array}{l}
\left|\rho_{0 K p} c_{4 p}-\rho_{0 K q} c_{4 q}\right|-2 f^{p q} \leq 0 \\
\left|\rho_{0 K p} s_{4 p}-\rho_{0 K q} s_{4 q}\right|-2 f^{p q} \leq 0 \\
\left|\rho_{1 p} c_{2 p}-\rho_{1 q} c_{2 q}\right|-2 f^{p q} \leq 0 \\
\left|\rho_{1 p} s_{2 p}-\rho_{1 q} s_{2 q}\right|-2 f^{p q} \leq 0
\end{array}\right.
$$

where

$$
f^{p q}:=\frac{\left|n_{p}-n_{q}\right|}{\max \left(n_{p}, n_{q}\right)}, \quad c_{\beta \gamma}:=\cos \left(\beta \phi_{1 \gamma} \frac{\pi}{2}\right), \quad s_{\beta \gamma}:=\sin \left(\beta \phi_{1 \gamma} \frac{\pi}{2}\right), \quad \beta=2,4, \quad \gamma=p, q .
$$

Inequalities (31) must be imposed for each couple of adjacent panels. Whatsoever, Eqs. (31) are not everywhere differentiable; a differentiable approximation should, hence, be considered. In particular, one may pose (Ramirez et al., 2014; Rudin, 1976)

$$
\begin{aligned}
\left|n_{p}-n_{q}\right| & =\lim _{\epsilon \rightarrow 0^{+}} \sqrt{\left(n_{p}-n_{q}\right)^{2}+\epsilon} \\
\max \left(n_{p}, n_{q}\right) & =\left\|\left\{n_{p}, n_{q}\right\}\right\|_{\infty}:=\lim _{m \rightarrow \infty}\left(n_{p}^{m}+n_{q}^{m}\right)^{\frac{1}{m}} .
\end{aligned}
$$

Let $\mathbf{g}$ be the vector collecting the four inequalities of Eq. (31) for each of the $n_{C}$ couples of adjacent laminates. In place of considering $4 n_{C}$ constraints, a unique equivalent constraint can be considered:

$$
g_{B}=\mathrm{LSE}_{\alpha}(\mathbf{g}) \leq 0, \quad \alpha>>1
$$

and

$$
\left(\nabla_{\boldsymbol{\xi}} g_{\text {blend }}\right)_{j}:=\frac{\partial g_{B}}{\partial \xi_{j}}=\frac{\sum_{i=1}^{4 n_{C}} e^{\alpha g_{i}} \frac{\partial g_{i}}{\partial \xi_{j}}}{\sum_{i=1}^{4 n_{C}} e^{\alpha g_{i}}}, \quad \alpha>>1, \quad j=1, \ldots, n_{\mathrm{vars}}
$$

It is noteworthy that the effect of $m$ can be disregarded when compared to the effect of $\alpha$ in Eq. (35). As an example, only the expression of the gradient for the first expression in Eq. (31) is derived. Remembering Eq. (33), the first inequality in Eq. (31) reads:

$$
\begin{aligned}
g_{B 1}^{p q} & :=\sqrt{\left(\rho_{0 K p} c_{4 p}-\rho_{0 K q} c_{4 q}\right)^{2}+\epsilon}-2\left(n_{p}^{m}+n_{q}^{m}\right)^{-\frac{1}{m}} \sqrt{\left(n_{p}-n_{q}\right)^{2}+\epsilon} \leq 0, \\
m & >>1, \quad \epsilon<<1 .
\end{aligned}
$$


After simple algebraic passages, non-zero derivatives are:

$$
\begin{aligned}
& \frac{\partial g_{B 1}^{p q}}{\partial n_{p}}=-2 \frac{\left(n_{p}-n_{q}\right)}{\left(n_{p}^{m}+n_{q}^{m}\right)^{\frac{1}{m}} \sqrt{\left(n_{p}-n_{q}\right)^{2}+\epsilon}}+2 \frac{n_{p}^{m-1}}{\left(n_{p}^{m}+n_{q}^{m}\right)^{\frac{1}{m}+1}} \sqrt{\left(n_{p}-n_{q}\right)^{2}+\epsilon}, \\
& \frac{\partial g_{B 1}^{p q}}{\partial n_{q}}=+2 \frac{\left(n_{p}-n_{q}\right)}{\left(n_{p}^{m}+n_{q}^{m}\right)^{\frac{1}{m}} \sqrt{\left(n_{p}-n_{q}\right)^{2}+\epsilon}}+2 \frac{n_{q}^{m-1}}{\left(n_{p}^{m}+n_{q}^{m}\right)^{\frac{1}{m}+1}} \sqrt{\left(n_{p}-n_{q}\right)^{2}+\epsilon} \\
& \frac{\partial g_{B 1}^{p q}}{\partial \rho_{0 K p}}=\frac{\left(\rho_{0 K p} c_{4 p}-\rho_{0 K q} c_{4 q}\right) c_{4 p}}{\sqrt{\left(\rho_{0 K p} c_{4 p}-\rho_{0 K q} c_{4 q}\right)^{2}+\epsilon}}, \\
& \frac{\partial g_{B 1}^{p q}}{\partial \rho_{0 K q}}=-\frac{c_{4 q}}{c_{4 p}} \frac{\partial g_{B 1}^{p q}}{\partial \rho_{0 K p}} \\
& \frac{\partial g_{B 1}^{p q}}{\partial \phi_{1 p}}=-2 \pi \frac{\left(\rho_{0 K p} c_{4 p}-\rho_{0 K q} c_{4 q}\right) \rho_{0 K p} s_{4 p}}{\sqrt{\left(\rho_{0 K p} c_{4 p}-\rho_{0 K q} c_{4 q}\right)^{2}+\epsilon}} \\
& \frac{\partial g_{B 1}^{p q}}{\partial \phi_{1 q}}=-\frac{\rho_{0 K q} s_{4 q} \partial g_{B 1}^{p q}}{\rho_{0 K p} s_{4 p}} \frac{\partial \phi_{1 p}}{\epsilon} \\
& \epsilon<<1, \quad m>>1 .
\end{aligned}
$$

Parameter $\alpha$ has been set as follows:

$$
\alpha(\operatorname{Var}(\mathbf{g}))= \begin{cases}500 & \text { if } \operatorname{Var}(\mathbf{g}) \leq 0.001 \\ 300 & \text { if } 0.001<\operatorname{Var}(\mathbf{g}) \leq 0.5 \\ 200 & \text { if } \operatorname{Var}(\mathbf{g})>0.5\end{cases}
$$

High values of $\alpha$ are due to the small variance of vector $\mathbf{g}$. Indeed, for very small values of $\operatorname{Var}(\mathbf{g})$, parameter $\alpha$ should take a very large value, which may lead to numerical overflows. In the limit case wherein the design variables are the same between adjacent panels, blending constraints assumes value close to zero (but not exactly zero, because of smoothing approximations). However, since $\mathrm{LSE}_{\alpha}$ approximates by excess the maximum, it can result in a violated constraint. To resolve this paradox, a correction is introduced only for very small values of $\operatorname{Var}(\mathbf{g})$. Considering Eq. (8), after few passages, the following inequality can be obtained:

$$
\max \mathbf{g}-\frac{1}{\alpha} \ln (n) \leq \mathrm{LSE}_{\alpha}(\mathbf{g})-\frac{1}{\alpha} \ln (n-\ln (n)) \leq \max \mathbf{g} .
$$

Therefore, only in the case of very small $\operatorname{Var}(\mathbf{g})$, the quantity $\operatorname{LSE}_{\alpha}(\mathbf{g})-\frac{1}{\alpha} \ln (n-\ln (n))$ (which is an underestimation) replaces Eq. (31). This occurs systematically when $\alpha$ assumes a large value.

\subsection{Global-Local Modelling Approach and Buckling Strength Requirement}

In order to asses correctly local phenomena, such as instabilities, a GL approach has been implemented. Three are the main issues connected with the GL approach:

1. the automatic detection of some zones of interest (ZOIs) which are likely to be critical in terms of buckling strength;

2. the automatic generation of a refined LFEM;

3. the correct transfer of loads from the GFEM into the LFEM as suitable BCs.

When using a deterministic optimisation algorithm, two main challenges arise for the analytic derivation of the buckling eigenvalue sensitivity: (1) the eigenvalue buckling analysis carried out on the LFEM is a pure Dirichlet problem, where only non-trivial BCs in terms of generalised displacements are assigned; (2) the BCs imposed on the LFEM are strictly related to the displacement field resulting 
from the GFEM, thus coupling effects between the GFEM and the LFEM must be integrated into the analytical expression of the gradient of the buckling factor resulting from the LFEM.

The buckling constraint can be formulated as:

$$
g_{\text {buck }}=1-\lambda \leq 0,
$$

where $\lambda$ is the first buckling factor of the associated eigenvalue problem

$$
\left(\mathbf{K}^{b}+\lambda \mathbf{K}_{\sigma}^{b}\right) \boldsymbol{\psi}^{b}=\mathbf{0},
$$

where $\mathbf{K}^{b}$ is the reduced stiffness matrix of the LFEM, $\mathbf{K}_{\sigma}^{b}$ is the reduced geometric stiffness matrix of the LFEM, $\boldsymbol{\psi}$ is the reduced eigenvector associated to $\lambda$. By deriving Eq. (40) with respect to the design variables, one obtains:

$$
\left(\nabla_{\xi} g_{\text {buck }}\right)_{j}:=\frac{\partial g_{\text {buck }}}{\partial \xi_{j}}=-\frac{\partial \lambda}{\partial \xi_{j}} .
$$

Appendix D is dedicated to the derivation of the analytical form of Eq. (42).

\subsection{The Non-Linear Programming Problem Formulation}

The first-level problem aims at determining the optimal distribution of PPs and numbers of plies in the structure, minimising the structure mass by satisfying, simultaneously, the design requirements discussed in the above subsections. The objective function can be formulated as follows:

$$
\Phi(\boldsymbol{\xi}):=\frac{m_{0}+\sum_{i=1}^{n_{\mathrm{pan}}} A_{i} n_{0 i} n_{\mathrm{ref}} t_{\mathrm{ply}} \rho_{\mathrm{ply}}}{m_{\mathrm{ref}}}
$$

where $m_{0}$ is the mass of the part of the structure which remains unchanged during the optimisation, $m_{\text {ref }}$ is a reference mass, $A_{i}, n_{0} i$ are the area and the dimensionless number of plies of the $i$-th region, respectively, $\rho_{\text {ply }}$ is the density of the single ply. Formally, the optimisation problem can be stated as a constrained non-linear programming problem (CNLPP) as:

$$
\begin{aligned}
& \min _{\boldsymbol{\xi}} \Phi(\boldsymbol{\xi}), \quad \text { subject to } \\
& \mathbf{K u}-\mathbf{f}=\mathbf{0}, \\
& \mathbf{K}^{b} \mathbf{u}^{b}+\mathbf{K}_{\mathrm{BC}}^{b} \mathbf{P u}=\mathbf{0}, \\
& g_{i}(\boldsymbol{\xi}) \leq 0, i=\text { feas, disp, TH, blend, buck, } \\
& \boldsymbol{\xi}_{\mathrm{lb}} \leq \boldsymbol{\xi} \leq \boldsymbol{\xi}_{\mathrm{ub}} .
\end{aligned}
$$

Table 2 reports lower and upper bounds for the considered design variables. The number of plies, which is a discrete variable, is assumed as a continuous one; at the end of the optimisation, the final value will be rounded.

Table 2: Design space for problem (44)

\begin{tabular}{ccc} 
Variable* $^{*}$ & lower bound & upper bound \\
\hline$n_{0}^{j}$ & 0.14 & 1 \\
$\rho_{0 K}^{j}$ & -1 & 1 \\
$\rho_{1}^{j}$ & 0 & 1 \\
$\phi_{1}^{j}$ & -1 & 1 \\
\hline${ }^{*} j=1, \ldots, n_{\text {pan }}$ &
\end{tabular}




\subsection{Numerical Strategy}

Problem (44) is a non-convex CNLPP in terms of both geometrical and mechanical design variables. The non-convexity is mainly due to the buckling eigenvalue as well as to strength and blending design requirements.

The solution search is performed via the active-set algorithm of the fmincon family, available in the Optimization Toolbox of MATLAB ${ }^{\circledR}$ (The MathWork Inc., 2011). The parameters tuning the behaviour of the algorithm have been kept to their default values, as summarised in Table 3. Of course, the strategy architecture is not affected by the particular choice of the deterministic algorithm. Sound alternatives are available in well-established open-source libraries, e.g. the Sequential Least Squares Programming (SLSQP) algorithm available in the Scipy package (Python) or the Interior Point OPTimizer (IPOPT) available in the PyOpt package (Python). In any case, the parameters governing the behaviour of the algorithm can be left unchanged with respect to the standard default values, as indicated in Table 3 about the fmincon parameters.

Table 3: fmincon parameters

\begin{tabular}{cc} 
Parameter & Value \\
\hline Solver & active-set \\
Maximum number of objective function evaluations & $100 \times n_{\text {vars }}$ \\
Maximum number of iterations & 1000 \\
Tolerance on objective function & $1 \times 10^{-6}$ \\
Tolerance on constraints & $1 \times 10^{-6}$ \\
Tolerance on input variables change & $10^{-6}$ \\
Tolerance on gradient norm of the Lagrange's function & $10^{-6}$ \\
\hline
\end{tabular}

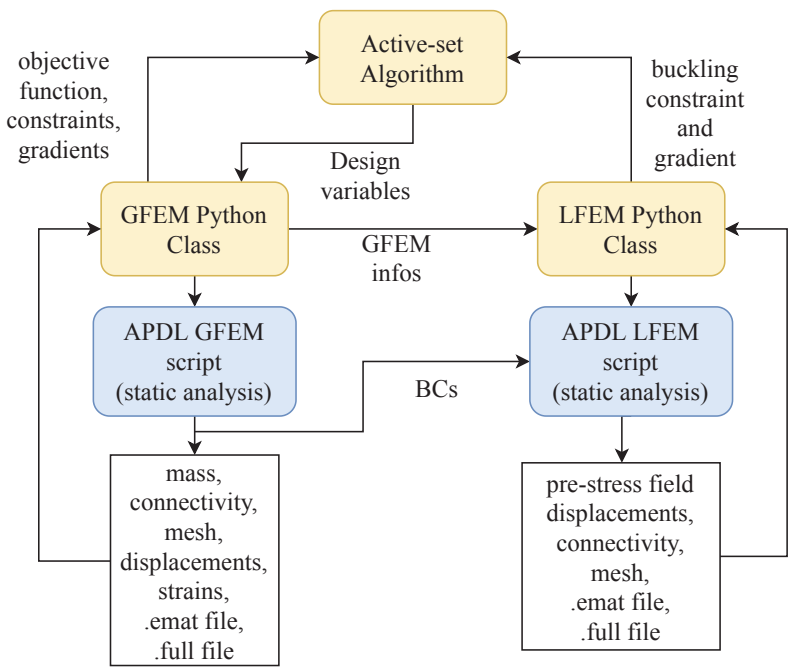

Figure 3: Workflow of the numerical strategy

Figure 3 shows the work-flow of the numerical strategy for the resolution of the first-level problem. The MATLAB ${ }^{\circledR}$ script invokes, at each iteration, the Python routines controlling the generation of the FE models (both global and local ones) used to assess the objective function and the optimisation constraints. Both the GFEM and the LFEMs are built in the ANSYS ${ }^{\circledR}$ environment through suitable ANSYS ${ }^{\circledR}$ Parametric Design Language (APDL) scripts. For each iteration, a new set of design variables $\boldsymbol{\xi}$ is passed from MATLAB ${ }^{\circledR}$ to a Python Class which represents the GFEM Object which invokes the APDL script generating the GFEM. Then, a static analysis is performed on the GFEM, and some fundamental information such as connectivity, nodes coordinates, displacement and strain fields, are passed to the GFEM Object (by means of .emat and .full files generated from ANSYS ${ }^{\circledR}$ at the end of the analysis). Subsequently, the GFEM Object evaluates feasibility, blending, maximum displacement and maximum strain constraints, together with their gradients. After this phase, the 
GFEM Object creates a new Object: the LFEM. The LFEM Object inherits some useful GFEM information (connectivity, etc.) and calls an APDL script generating the LFEM for the most critical ZOI. Once BCs extracted from the GFEM are applied to the LFEM, a static analysis with pre-stress effects is solved on the LFEM. Some information such as pre-stress displacements, connectivity, etc. are passed back to the LFEM Object. Matrix $\mathbf{K}_{\sigma}^{b}$ is assembled by the LFEM Class, according to the expression presented in Appendix D. The LFEM Class also solves the eigenvalue buckling problem on the LFEM via scipy.sparse.linalg.eigsh routine, based on the ARPACK software and the Implicitly Restarted Lanczos Method (Lehoucq et al., 1998). Finally, the buckling constraint of Eq. (42) and its gradient are evaluated (according to the procedure detailed in Appendix D).

The objective function and the optimisation constraints, with the related gradients, are then passed to the optimisation algorithm. The loop is repeated until one of the convergence criteria, listed in Table 3, is satisfied.

The choice of assembling the LFEM geometric stiffness matrix in Python environment, instead of just exploiting the .emat file provided by ANSYS ${ }^{\circledR}$, is due to the fact that ANSYS ${ }^{\circledR}$ software makes use of some correction factors, not provided in the ANSYS ${ }^{\circledR}$ manual Ansys ${ }^{\circledR}$ (2013), which depend on the element geometric and material properties. Moreover, as detailed in Appendix D, in this work, an alternative (general) definition of the element geometric stiffness matrix (different from that used in classic FE codes) has been used in order to derive an efficient closed-form expression of the buckling factor gradient. All these details are of paramount importance for a correct assessment of the buckling factor gradient and represent the reason at the basis of using Python instead of ANSYS ${ }^{\circledR}$ for the resolution of the eigenvalue problem.

\section{The Global/Local Finite Element modelling Approach}

As stated above, the FE models integrated in the optimisation process are based on a GL modelling approach, more precisely on the sub-modelling technique, see the works by Sun and Mao (1988); Mao and Sun (1991); Whitcomb (1991) for more details on this topic. According to the strategy discussed in Izzi et al. (2020), two different FE models are created: the GFEM for the assessment of the global behaviour of the wing-box, and a refined LFEM in order to properly evaluate the local responses (in this case the first buckling factor). The LFEM is created only at the most critical ZOI (from a buckling strength perspective), which is represented by the dorsal panels composing the wing-box, as illustrated in Fig. 5. Therefore, inasmuch as for the simple benchmark considered in this study the most critical ZOI is known a-priori, there is no need to introduce suitable criteria for automatically identifying the most critical ZOIs, as done in Izzi et al. (2020). Nevertheless, as deeply discussed in Appendix D, the coupling effects between GFEM and LFEM are still important when evaluating the gradient of the buckling factor for optimisation purposes.

Both GFEM and LFEMs are fully parametric and are built using the commercial FE code ANSYS ${ }^{\circledR}$. The GFEM is illustrated in Fig.4. The root section is clamped, whilst external forces $F_{1}=90009.77 \mathrm{~N}, F_{2}=F_{3}=187888.44 \mathrm{~N}, F_{4}=380176.16 \mathrm{~N}$ are applied at the tip section nodes (from $\mathrm{B}$ to E) as illustrated in the same figure. The model is made of SHELL181 elements, which are based on Reissner-Mindlin kinematics (Reddy, 2003) and have four nodes with six DOFs per node. The GFEM is composed of about 560 elements and 3000 DOFs. The mesh size has been chosen after a sensitivity study (not reported here for the sake of brevity) in order to find a compromise between accuracy and computational cost.

The LFEM model is created to evaluate the first buckling load of the dorsal skin of the wing-box, as shown in Fig. 5, and it is generated via a dedicated APDL script. BCs on generalised displacements, extracted from the results of the global analysis and illustrated in Fig. 5, are imposed to all the boundary nodes belonging to the skin of each dorsal panel (representing the connections between ribs, spars and stringers). The LFEM is made of SHELL181 elements, and the mesh is finer than the dorsal skin of the GFEM. Therefore, BCs extracted from the boundary nodes of each panel composing the GFEM are properly interpolated during the transfer from the GFEM to the LFEM (by using the element shape functions). To set a suitable value of the element size of the LFEM, a sensitivity analysis of the first buckling factor to this parameter has been performed also in this case (and it is 


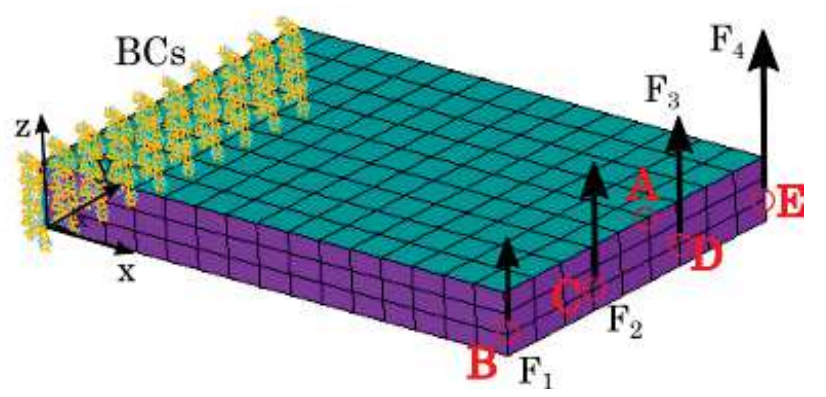

Figure 4: GFEM and the related BCs

not reported here for the sake of brevity). As a result, the LFEM is characterised by approximately 490 elements and 3200 DOFs.

With such a mesh, the computational cost is approximately of $30 \mathrm{~s}$ for each iteration when four cores of a machine with an Intel ${ }^{\circledR}$ Xeon E5-2697v2 processor $(2.70-3.50 \mathrm{GHz})$ are dedicated to the ANSYS ${ }^{\circledR}$ solver.

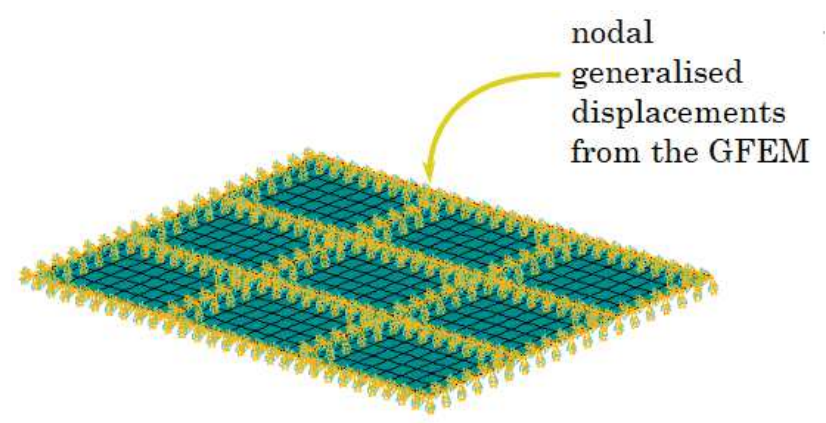

Figure 5: LFEM and the related BCs

\section{$5 \quad$ Numerical Results}

Since the objective function of Eq.(43) is an hyper-plane in the $\mathbb{R}^{n_{\text {vars }}}$ space, due to its linearity with respect to geometrical variables, it is expected that the optimal solution exists, and that it is located at the boundary of the feasible region. Because of the high complexity of problem (44), the choice of a feasible starting guess is of paramount importance. In the following, three test cases are discussed, showing the importance of the buckling constraint and the differences in the optimised solution when neglecting the out-of-plane shear contribution in the expression of $\mathbf{K}_{\sigma}^{b}$.

The reference value of the mass, i.e. $m_{\text {ref }}=314.12 \mathrm{~kg}$, is taken from Liu et al. (2011). The best known value for the optimised mass is $276.47 \mathrm{~kg}$, corresponding to a normalised value of 0.88 , obtained via a meta-heuristic algorithm in Panettieri et al. (2019), even though a slightly different formulation of problem (44).

\subsection{Case 1: Optimisation Without Buckling Strength Requirement}

Firstly, problem (44) has been solved without considering the design requirement on the first buckling factor evaluated on the LFEM. The starting point has been set to $n_{0}^{j}=0.4, \rho_{0 K}^{j}=\rho_{1}^{j}=\phi_{1}^{j}=0.0$, $j=1, \ldots, n_{\text {pan }}$ (see Fig. 2). Such a starting point, in the feasible domain, represents a wing-box structure composed of isotropic laminates because the anisotropic moduli are null.

The algorithm converges towards a feasible solution after 400 iterations. The optimal value of the design variables is shown in Fig. 6 ( $n_{0}$ in blue, $\rho_{0 K}$ in red, $\rho_{1}$ in green, $\phi_{1}$ in black), whilst Table 5 reports the numerical values. Due to the absence of an optimisation constraint on the first buckling load, the resulting structural mass takes low value: the objective function is 0.5603 , corresponding to 


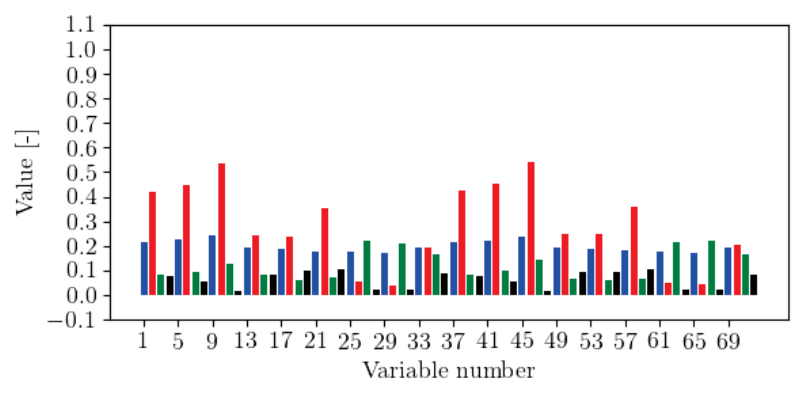

Figure 6: Optimal design variables values for Case 1

an overall mass of $176 \mathrm{~kg}$, as indicated in Table 4 . It is noteworthy that the solution is almost symmetric between dorsal and ventral panels. The active constraint is the maximum strain, as reported in Table 4. This confirms the expectations stated at the preamble of this section: the solution lies on the boundary of the feasible domain.

\subsection{Effect of the Buckling Strength Requirement on the Optimised Solution}

\subsubsection{Case 2: Only Membrane Contribution in $\mathbf{K}_{\sigma}^{b}$}

The introduction of the design requirement on the first buckling factor introduces a strong non-linear behaviour into the optimisation problem formulation. This non-linear behaviour is due, on the one hand, to the assessment of the buckling factor on a refined LFEM, and, on the other hand, to the definition of the structure geometric stiffness matrix $\mathbf{K}_{\sigma}^{b}$ as a function of the design variables of the problem at hand. In a first time, the effect of only the membrane contribution in the definition of $\mathbf{K}_{\sigma}^{b}$ (i.e. matrices $\overline{\mathbf{K}}_{1}, \overline{\mathbf{K}}_{2}, \overline{\mathbf{K}}_{3}$ in Appendix D) on the optimised solution is considered. In order to help

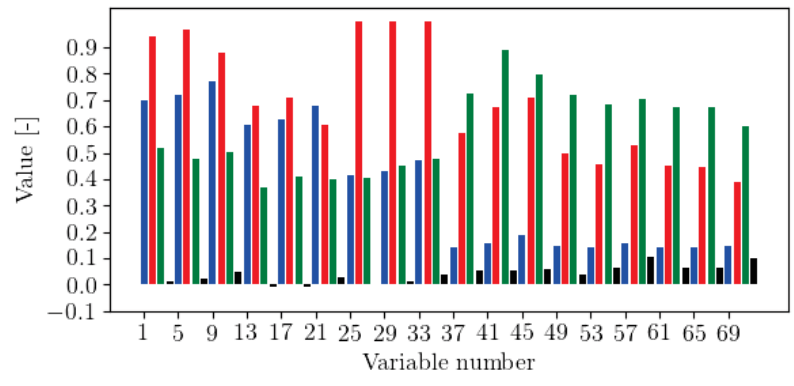

Figure 7: Optimal design variables values for Case 2

the convergence, the starting point has been set to $n_{0}^{j}=0.8, \rho_{0 K}^{j}=\rho_{1}^{j}=\phi_{1}^{j}=0.0, j=1, \ldots, 9$ for dorsal panels and to $n_{0}^{j}=0.4, \rho_{0 K}^{j}=\rho_{1}^{j}=\phi_{1}^{j}=0.0, j=10, \ldots, n_{\text {pan }}$ for the ventral ones (see Fig. 2). This starting point, which lies in the feasible domain, is more likely to be closer to the optimal point. Moreover, also in this case, the starting point is an isotropic solution.

The algorithm converges after 500 iterations. Although the starting point is inside the feasible domain, the algorithm experiences many difficulties in find the good path, due to the strong non-linear nature of the problem. The objective function value is 0.8440 , corresponding to a mass equal to $265.12 \mathrm{~kg}$. As expected, the mass is slight lower then the value reported in Panettieri et al. (2019), because of the neglected out-of-plane shear contribution in the expression of the geometric stiffness matrix. As in Panettieri et al. (2019), the solution lies on the boundary of the buckling constraint.

The optimal value of the design variables is illustrated in Fig. 7 ( $n_{0}$ in blue, $\rho_{0 K}$ in red, $\rho_{1}$ in green, $\phi_{1}$ in black), whilst the numerical values are reported in Table 5. The objective function and the optimisation constraints are listed in Table 4. A quick glance to this result highlights an enhanced exploitation of the anisotropy, especially for dorsal panels (variables 1 up to 36), if compared to the optimal solution of case 1. 


\subsubsection{Case 3: Membrane and Out-of-Plane Shear Contributions in $\mathbf{K}_{\sigma}^{b}$}

This case considers both the membrane and the out-of-plane shear contributions in the expression of $\mathbf{K}_{\sigma}^{b}$ (see Appendix D). This formulation is really close to that implemented in ANSYS ${ }^{\circledR}$ software.

The starting point has been set to $n_{0}^{j}=0.8, \rho_{0 K}^{j}=\rho_{1}^{j}=\phi_{1}^{j}=0.0, j=1, \ldots, 9$ for dorsal panels and

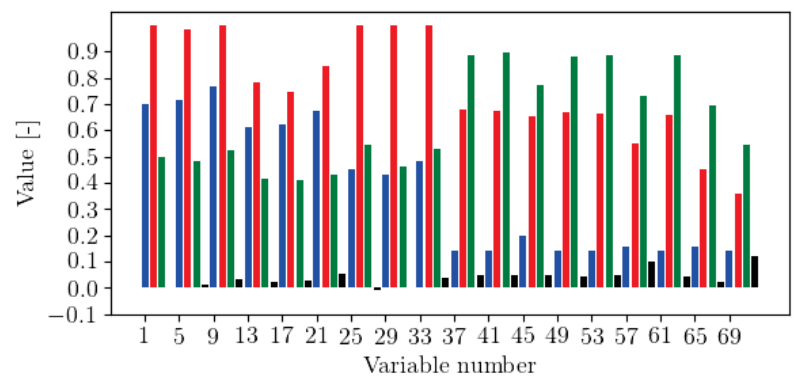

Figure 8: Optimal design variables values for Case 3

to $n_{0}^{j}=0.4, \rho_{0 K}^{j}=\rho_{1}^{j}=\phi_{1}^{j}=0.0, j=10, \ldots, n_{\text {pan }}$ for the ventral ones (see Fig. 2). Also in this case the starting point is an isotropic solution.

The algorithm converges after 400 iterations. The objective function value is 0.8461 , corresponding to an overall mass of $265.72 \mathrm{~kg}$. The optimal value of the design variables is shown in Fig. 8 and listed in Table 5. The values of the objective function and of the optimisation constraints are reported in Table 4. When compared to the solution of case 2 , this configuration is characterised by a more pronounced effect of the anisotropy on the buckling strength (especially in terms of $\rho_{0 K}$ and $\rho_{1}$ contributions) and by a different orientation of the main orthotropy axis (related to the value of the polar angle). However, since the problem is strongly non-convex in terms of the buckling factor requirement, both solutions are characterised by similar values of the mass and of the buckling factor constraint.

Table 4: Objective function for the optimised solution

\begin{tabular}{cccccccc} 
Case & Objective function & Mass $[\mathrm{kg}]$ & $g_{\text {feas }}$ & $g_{\text {blend }}$ & $g_{\text {TH }}$ & $g_{\text {disp }}$ & $g_{\text {buck }}$ \\
\hline Panettieri et al. (2019) & 0.88 & 276.47 & - & - & - & - & - \\
Case 1 & 0.5603 & 176 & -0.9215 & -0.0125 & $-6 \times 10^{-7}$ & -0.3202 & - \\
Case 2 & 0.8440 & 265.12 & -0.0897 & $-3 \times 10^{-5}$ & $-2 \times 10^{-5}$ & -0.4316 & $-7 \times 10^{-5}$ \\
Case 3 & 0.8461 & 265.77 & -0.0540 & $-1 \times 10^{-5}$ & $-6 \times 10^{-6}$ & -0.4322 & $-1 \times 10^{-5}$ \\
\hline
\end{tabular}

Table 5: Optimal value of design variables

\begin{tabular}{c|cccc|cccc|cccc} 
Panel & \multicolumn{9}{|c}{ Case 1} \\
\hline ID & $n_{0}$ & $\rho_{0 K}$ & $\rho_{1}$ & $\phi_{1}$ & $n_{0}$ & $\rho_{0 K}$ & $\rho_{1}$ & $\phi_{1}$ & $n_{0}$ & $\rho_{0 K}$ & $\rho_{1}$ & $\phi_{1}$ \\
\hline 1 & 0.2147 & 0.4171 & 0.0788 & 0.0757 & 0.7001 & 0.9423 & 0.5172 & 0.0144 & 0.6972 & 0.9999 & 0.4980 & 0.0020 \\
2 & 0.2237 & 0.4491 & 0.0943 & 0.0532 & 0.7208 & 0.9655 & 0.4782 & 0.0241 & 0.7159 & 0.9855 & 0.4846 & 0.0111 \\
3 & 0.2404 & 0.5361 & 0.1281 & 0.0142 & 0.7700 & 0.8791 & 0.5016 & 0.0498 & 0.7655 & 1.0000 & 0.5247 & 0.0316 \\
4 & 0.1941 & 0.2394 & 0.0833 & 0.0805 & 0.6065 & 0.6783 & 0.3666 & -0.0086 & 0.6120 & 0.7837 & 0.4157 & 0.0239 \\
5 & 0.1893 & 0.2349 & 0.0583 & 0.0958 & 0.6287 & 0.7077 & 0.4110 & -0.0075 & 0.6240 & 0.7463 & 0.4112 & 0.0291 \\
6 & 0.1782 & 0.3547 & 0.0714 & 0.1039 & 0.6760 & 0.6056 & 0.3973 & 0.0266 & 0.6729 & 0.8434 & 0.4293 & 0.0531 \\
7 & 0.1758 & 0.0543 & 0.2202 & 0.0182 & 0.4164 & 1.0000 & 0.4044 & 0.0001 & 0.4493 & 1.0000 & 0.5426 & -0.0104 \\
8 & 0.1712 & 0.0366 & 0.2109 & 0.0197 & 0.4300 & 1.0000 & 0.4490 & 0.0099 & 0.4293 & 1.0000 & 0.4601 & 0.0032 \\
9 & 0.1924 & 0.1921 & 0.1661 & 0.0860 & 0.4742 & 1.0000 & 0.4789 & 0.0393 & 0.4828 & 1.0000 & 0.5285 & 0.0379 \\
\hline 10 & 0.2136 & 0.4243 & 0.0817 & 0.0750 & 0.1400 & 0.5765 & 0.7271 & 0.0518 & 0.1400 & 0.6775 & 0.8847 & 0.0460 \\
11 & 0.2224 & 0.4540 & 0.0962 & 0.0537 & 0.1569 & 0.6728 & 0.8896 & 0.0540 & 0.1400 & 0.6751 & 0.8941 & 0.0463 \\
12 & 0.2388 & 0.5391 & 0.1400 & 0.0146 & 0.1882 & 0.7071 & 0.7977 & 0.0565 & 0.1988 & 0.6505 & 0.7723 & 0.0459 \\
13 & 0.1919 & 0.2456 & 0.0626 & 0.0937 & 0.1444 & 0.4969 & 0.7190 & 0.0394 & 0.1399 & 0.6668 & 0.8815 & 0.0447 \\
14 & 0.1892 & 0.2457 & 0.0620 & 0.0936 & 0.1400 & 0.4570 & 0.6813 & 0.0637 & 0.1400 & 0.6645 & 0.8843 & 0.0467 \\
15 & 0.1787 & 0.3562 & 0.0651 & 0.1018 & 0.1548 & 0.5285 & 0.7068 & 0.1063 & 0.1553 & 0.5504 & 0.7293 & 0.0986 \\
16 & 0.1736 & 0.0463 & 0.2156 & 0.0202 & 0.1400 & 0.4527 & 0.6744 & 0.0631 & 0.1400 & 0.6582 & 0.8853 & 0.0434 \\
17 & 0.1703 & 0.0399 & 0.2211 & 0.0199 & 0.1400 & 0.4447 & 0.6748 & 0.0641 & 0.1548 & 0.4525 & 0.6936 & 0.0248 \\
18 & 0.1925 & 0.2011 & 0.1667 & 0.0839 & 0.1468 & 0.3883 & 0.6004 & 0.1000 & 0.1400 & 0.3575 & 0.5462 & 0.1215 \\
\hline
\end{tabular}


As an example, Figs. 9a, 10a, 11a show the first component of the membrane tensor A, whilst Figs. 9b, 10b, 11b illustrate the first component of the out-of-plane shear tensor $\mathbf{H}$, for panels 1, 6, 7, respectively. The square mark shows the relative angle $\Phi_{1}^{A^{*}}$. It is evident the impact of considering

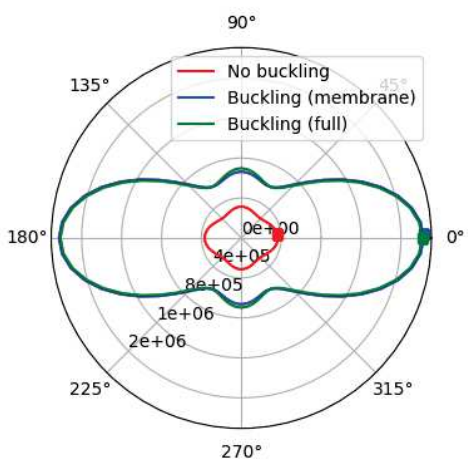

(a) $A_{11}$ coefficient

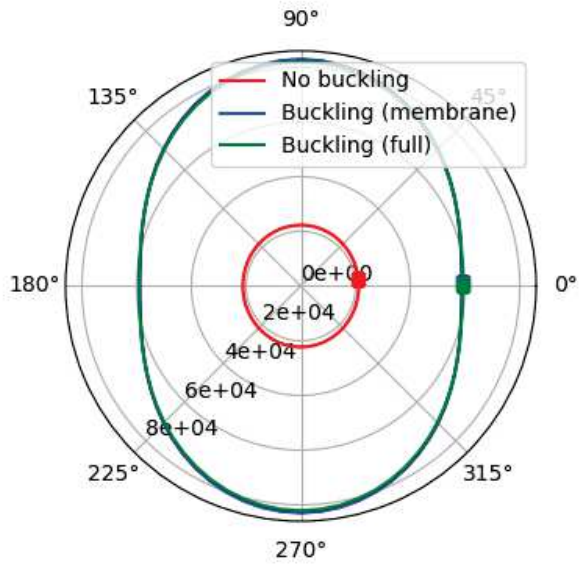

(b) $H_{11}$ coefficient

Figure 9: Polar diagram of the first component of matrices $\mathbf{A}$ and $\mathbf{H}$ for panel $\mathrm{n}^{\circ} 1$

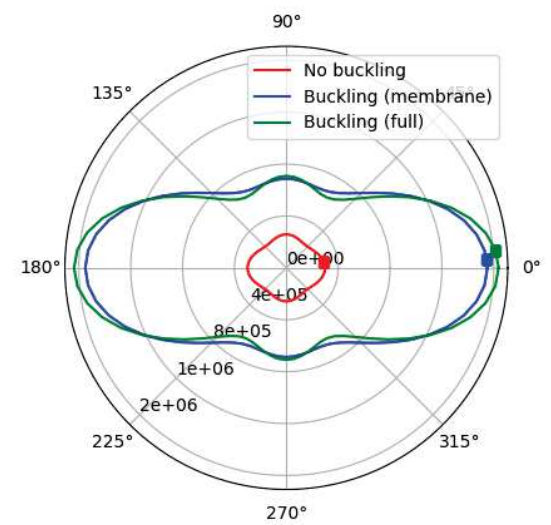

(a) $A_{11}$ coefficient

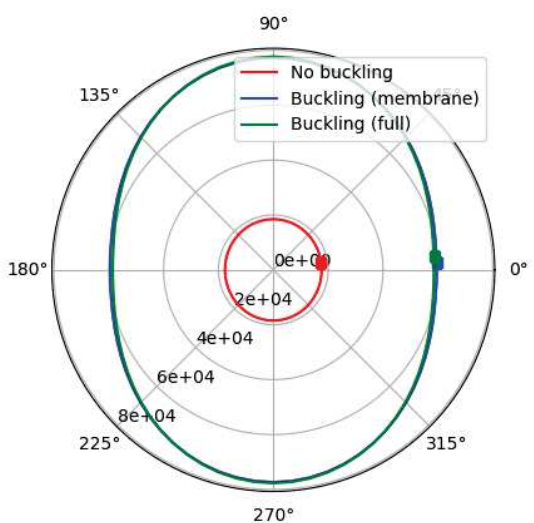

(b) $H_{11}$ coefficient

Figure 10: Polar diagram of the first component of matrices $\mathbf{A}$ and $\mathbf{H}$ for panel $\mathrm{n}^{\mathrm{o}} 6$

the buckling strength requirement on the optimised value of the laminate PPs: the polar diagram of the laminate stiffness matrices changes considerably for the considered cases. Conversely, the effect of the membrane and shear contributions involved in the definition of the geometric stiffness matrix it is more pronounced only for panels 6 and 7, as it can be inferred from Table 5 and Figs. 10 and 11.

\section{Conclusions}

A general theoretical framework for the deterministic optimisation of composite structures has been presented in this work. The proposed approach is based on the polar formalism for the description of the anisotropy and on the FSDT to represent the kinematics of each laminate composing the structure at the macroscopic scale. A general GL modelling approach, based on the sub-modelling technique, is integrated into the MS2LOS of composite structures developed by Montemurro and his co-workers. The resulting methodology, called GL-MS2LOS, aims at proposing a very general formulation of the design problem, without introducing simplifying hypotheses on the laminate stack and by considering, as design variables, the full set of geometric and mechanical parameters defining the behaviour of 


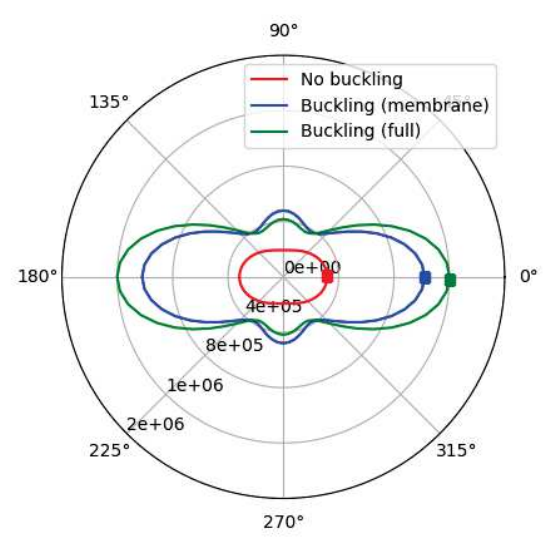

(a) $A_{11}$ coefficient

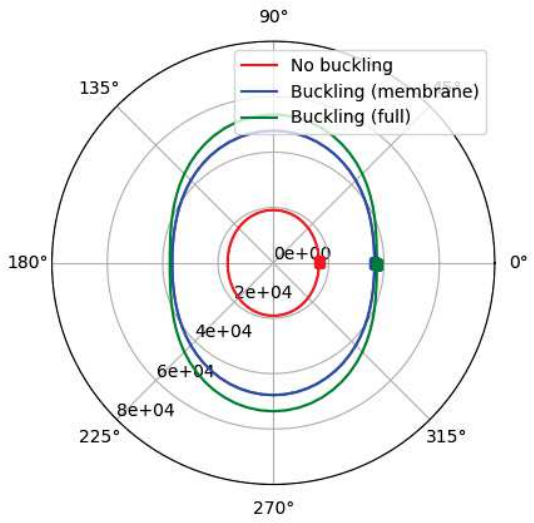

(b) $H_{11}$ coefficient

Figure 11: Polar diagram of the first component of matrices $\mathbf{A}$ and $\mathbf{H}$ for panel $\mathrm{n}^{\mathrm{o}} 7$

the composite structure at each pertinent scale. In this work, only the first-level problem (laminate macroscopic scale) is considered.

In this background, a variety of constraints has been integrated in the problem formulation and expressed in the PPs space; moreover, the analytic form of their gradients has been derived. In particular, the GL modelling approach has been integrated in the formulation of the related optimisation problem, in order to assess the physical responses involved at different levels. Of course, the coupling effect between global and local models on the gradient of the physical responses, evaluated on the refined local model, has been taken into account.

The effectiveness of the GL-MS2LOS has been tested on a numerical benchmark, taken from the literature, dealing with the least-weight design of a simplified wing-box structure: the numerical results, in terms of optimised solutions, are very encouraging.

As far as prospects of this work are concerned, the proposed framework can be extended to more complex structures, such as aeronautical ones, considering different local models and numerous load cases. Furthermore, there is still a lack of an effective strategy for the resolution of the second-level problem of the MS2LOS, in order to recover blended stacking sequences for the whole structure, with minor apriori hypotheses on the nature of the stacks.

Research is ongoing in this sense.

\section{Replication of Results}

Sufficient details of the implemented approach have been provided in this paper. Authors are confident that the results can be reproduced. Readers interested in the Python or ANSYS ${ }^{\circledR}$ APDL scripts are encouraged to contact the corresponding author via email.

\section{Conflict of Interest Statement}

On behalf of all authors, the corresponding author states that there is no conflict of interest.

\section{Acknowledgements}

This paper presents part of the activities carried out within the research project PARSIFAL ("PrandtlPlane ARchitecture for the Sustainable Improvement of Future AirpLanes"), which has been funded by the European Union under the Horizon 2020 Research and Innovation Program (Grant Agreement n.723149). 


\section{A Analytic Expression of Laminate Stiffness Matrices Gradient}

Under the hypothesis of orthotropic laminate, the expression of the homogenised membrane stiffness matrix in terms of the dimensionless PPs reads:

$$
\begin{aligned}
\mathbf{A}^{*} & =\underbrace{\left[\begin{array}{ccc}
T_{0}+2 T_{1} & -T_{0}+2 T_{1} & 0 \\
& T_{0}+2 T_{1} & 0 \\
\operatorname{sym} & & T_{0}
\end{array}\right]}_{\mathbf{A}_{0}^{*}}+R_{0 K}^{A^{*}} \underbrace{\left[\begin{array}{ccc}
c_{4} & -c_{4} & s_{4} \\
& c_{4} & -s_{4} \\
\operatorname{sym} & & -c_{4}
\end{array}\right]}_{\mathbf{A}_{1}^{*}}+R_{1}^{A^{*}} \underbrace{\left[\begin{array}{ccc}
4 c_{2} & 0 & 2 s_{2} \\
& -4 c_{2} & 2 s_{2} \\
\operatorname{sym} & 0
\end{array}\right]}_{\mathbf{A}_{2}^{*}} \\
& :=\mathbf{A}_{0}^{*}+R_{0} \rho_{0 K} \mathbf{A}_{1}^{*}+R_{1} \rho_{1} \mathbf{A}_{2}^{*},
\end{aligned}
$$

with

$$
c_{2}=\cos \pi \phi_{1}, \quad c_{4}=\cos 2 \pi \phi_{1}, \quad s_{2}=\sin \pi \phi_{1}, \quad s_{4}=\sin 2 \pi \phi_{1} .
$$

Similarly, matrix $\mathbf{H}^{*}$ can be decomposed as:

$$
\mathbf{H}^{*}=\underbrace{\left[\begin{array}{cc}
T & 0 \\
\operatorname{sym} & T
\end{array}\right]}_{\mathbf{H}_{0}^{*}}+R \rho_{1} \underbrace{\left[\begin{array}{cc}
c_{2}^{H^{*}} & s_{2}^{H^{*}} \\
\operatorname{sym} & -c_{2}^{H^{*}}
\end{array}\right]}_{\mathbf{H}_{1}^{*}}:=\mathbf{H}_{0}^{*}+R \rho_{1} \mathbf{H}_{1}^{*},
$$

where

$$
c_{2}^{H^{*}}=\cos 2 \Phi^{H^{*}}, \quad s_{2}^{H^{*}}=\sin 2 \Phi^{H^{*}}, \quad \Phi^{H^{*}}=\Phi+\Phi_{1}-\frac{\pi}{2} \phi_{1} .
$$

Since quasi-homogeneity holds, $\mathbf{B}^{*}=\mathbf{O}$ and $\mathbf{D}^{*}=\mathbf{A}^{*}$.

Let $h=t_{\mathrm{ply}} n_{0} n_{\mathrm{ref}}$, with reference to Eq. (4). Therefore, the following derivatives read:

$$
\begin{aligned}
\frac{\partial \mathbf{A}}{\partial n_{0}} & =\frac{h}{n_{0}} \mathbf{A}^{*}, \quad \frac{\partial \mathbf{A}}{\partial \rho_{0 K}}=h R_{0} \mathbf{A}_{1}^{*}, \quad \frac{\partial \mathbf{A}}{\partial \rho_{1}}=h R_{1} \mathbf{A}_{2}^{*}, \\
\frac{\partial \mathbf{A}}{\partial \phi_{1}} & =2 \pi h R_{0} \rho_{0 K}\left[\begin{array}{ccc}
-s_{4} & +s_{4} & c_{4} \\
& -s_{4} & -c_{4} \\
\operatorname{sym} & & +s_{4}
\end{array}\right]+\pi h R_{1} \rho_{1}\left[\begin{array}{ccc}
-4 s_{2} & 0 & 2 c_{2} \\
& +4 s_{2} & 2 c_{2} \\
\text { sym } & & 0
\end{array}\right] .
\end{aligned}
$$

Similarly, for matrix $\mathbf{D}$ and matrix $\mathbf{H}$ :

$$
\begin{aligned}
& \frac{\partial \mathbf{D}}{\partial n_{0}}=\frac{h^{2}}{4} \frac{\partial \mathbf{A}}{\partial n_{0}}, \quad \frac{\partial \mathbf{D}}{\partial \rho_{0 K}}=\frac{h^{2}}{12} \frac{\partial \mathbf{A}}{\partial \rho_{0 K}}, \quad \frac{\partial \mathbf{D}}{\partial \rho_{1}}=\frac{h^{2}}{12} \frac{\partial \mathbf{A}}{\partial \rho_{1}}, \quad \frac{\partial \mathbf{D}}{\partial \phi_{1}}=\frac{h^{2}}{12} \frac{\partial \mathbf{A}}{\partial \phi_{1}}, \\
& \frac{\partial \mathbf{H}}{\partial n_{0}}=\frac{h}{n_{0}} \mathbf{H}^{*}, \quad \frac{\partial \mathbf{H}}{\partial \rho_{0 K}}=\mathbf{O}, \quad \frac{\partial \mathbf{H}}{\partial \rho_{1}}=h R \mathbf{H}_{1}^{*}, \quad \frac{\partial \mathbf{H}}{\partial \phi_{1}}=-\pi h R \rho_{1}\left[\begin{array}{cc}
-s_{2}^{H^{*}} & c_{2}^{H^{*}} \\
\mathrm{sym} & +s_{2}^{H^{*}}
\end{array}\right] .
\end{aligned}
$$

Finally, for orthotropic quasi-homogeneous laminates, for the generic $\xi_{j}$ :

$$
\frac{\partial \mathbf{K}_{e}^{l a m}}{\partial \xi_{j}}=\operatorname{diag}\left(\frac{\partial \mathbf{A}_{e}}{\partial \xi_{j}}, \frac{\partial \mathbf{D}_{e}}{\partial \xi_{j}}, \frac{\partial \mathbf{H}_{e}}{\partial \xi_{j}}\right)
$$

\section{B Analytic Expression of Stiffness Matrix Gradient}

The unconstrained equilibrium system of the GFEM is of the form

$$
\hat{\mathbf{K}} \hat{\mathbf{u}}=\hat{\mathbf{f}}
$$

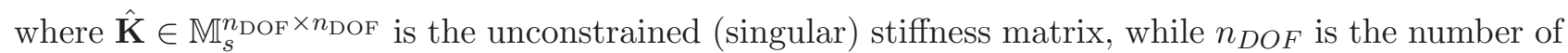
DOFs of the GFEM before the application of the BCs. 
Definition B.1 Given a matrix $M \in \mathbb{M}^{\mathrm{m} \times \mathrm{n}}$ and the two sets of positive natural numbers $R \subset\{i \mid 1 \leq$ $i \leq \mathrm{m}\}$ and $C \subset\{j \mid 1 \leq j \leq \mathrm{n}\}$, the operator $\mathfrak{R}(\boldsymbol{M}, R, C)$ returns the matrix obtained by suppressing the $i$-th row and the $j$-th column of $\boldsymbol{M}, \forall i \in R$ and $\forall j \in C$. Similarly, $\Re(\boldsymbol{v}, R)$ denotes the vector obtained by suppressing the $i$-th row of $\boldsymbol{v}, \forall i \in R$.

If BCs are of the type $u_{j}=0$ for $j \in I_{\mathrm{BC}} \subset\left\{i \mid i=1, \ldots, n_{\mathrm{DOF}}\right\}, \sharp I_{\mathrm{BC}}=n_{\mathrm{BC}}$, Eq. (B.1) can be transformed in a reduced problem of the form and size considered in Eq. (13) by posing $\mathbf{K}:=\mathfrak{R}\left(\hat{\mathbf{K}}, I_{\mathrm{BC}}, I_{\mathrm{BC}}\right), \mathbf{u}:=\mathfrak{R}\left(\hat{\mathbf{u}}, I_{\mathrm{BC}}\right)$ and $\mathbf{f}:=\mathfrak{R}\left(\hat{\mathbf{f}}, I_{\mathrm{BC}}\right)$.

The analytical form of $\partial \mathbf{K} / \partial \xi_{j}$ can be easily determined. In fact, the expression of $\hat{\mathbf{K}}$ is:

$$
\hat{\mathbf{K}}=\sum_{e=1}^{N_{e}} \hat{\mathbf{L}}_{e}^{\mathrm{T}} \int_{\Omega_{e}} \mathbf{B}_{e}^{\mathrm{T}} \mathbf{K}_{e}^{l a m} \mathbf{B}_{e} \mathrm{~d} \Omega_{e} \hat{\mathbf{L}}_{e}
$$

where $N_{e}$ is the number of elements of the GFEM, $\Omega_{e}$ is the integration domain for the $e$-th element, $\mathbf{B}_{e}$ is the operator defined in Eq. (26), $\mathbf{K}_{e}^{\text {lam }}$ is the element stiffness matrix defined in Eq. (2), expressed in the global frame of the GFEM, whilst $\hat{\mathbf{L}}_{e} \in \mathbb{M}^{24 \times n_{\mathrm{DOF}}}$ is a linear map $\hat{\mathbf{L}}_{e}: \hat{\mathbf{u}} \mapsto \mathbf{u}_{e}$. By deriving Eq. (B.2) with respect to the generic $\xi_{j}$, one obtains:

$$
\frac{\partial \hat{\mathbf{K}}}{\partial \xi_{j}}=\sum_{e=1}^{N_{e}} \hat{\mathbf{L}}_{e}^{\mathrm{T}} \int_{\Omega_{e}} \mathbf{B}_{e}^{\mathrm{T}} \frac{\partial \mathbf{K}_{e}^{l a m}}{\partial \xi_{j}} \mathbf{B}_{e} \mathrm{~d} \Omega_{e} \hat{\mathbf{L}}_{e}
$$

It follows that

$$
\frac{\partial \mathbf{K}}{\partial \xi_{j}}:=\mathfrak{R}\left(\frac{\partial \hat{\mathbf{K}}}{\partial \xi_{j}}, I_{\mathrm{BC}}, I_{\mathrm{BC}}\right) .
$$

\section{Analytic Expression of Laminate Strength Matrices Gradient}

Matrix $\mathbf{G}_{A}^{*}$ can be decomposed as:

$$
\begin{aligned}
\mathbf{G}_{A}^{*} & =\underbrace{\left[\begin{array}{ccc}
\Gamma_{0}+2 \Gamma_{1} & -\Gamma_{0}+2 \Gamma_{1} & 0 \\
& \Gamma_{0}+2 \Gamma_{1} & 0 \\
\operatorname{sym} & & \Gamma_{0}
\end{array}\right]}_{\mathbf{G}_{A 0}^{*}}+\Lambda_{0}^{G_{A}^{*}} \underbrace{\left[\begin{array}{ccc}
c_{4} & -c_{4} & s_{4} \\
& c_{4} & -s_{4} \\
\operatorname{sym} & & -c_{4}
\end{array}\right]}_{\mathbf{G}_{A 1}^{*}}+\Lambda_{1}^{G_{A}^{*}} \underbrace{\left[\begin{array}{ccc}
4 c_{2} & 0 & 2 s_{2} \\
& -4 c_{2} & 2 s_{2} \\
\operatorname{sym} & 0
\end{array}\right]}_{\mathbf{G}_{A 2}^{*}} \\
& :=\mathbf{G}_{A 0}^{*}+\rho_{0 K} \Lambda_{0} \mathbf{G}_{A 1}^{*}+\Lambda_{1} \rho_{1} \mathbf{G}_{A 2}^{*},
\end{aligned}
$$

with

$$
\begin{aligned}
c_{2} & =\cos 2\left(\frac{\pi}{2} \phi_{1}+\Omega_{1}-\Phi_{1}\right), \quad c_{4}=\cos 4\left(\frac{\pi}{2} \phi_{1}+\Omega_{0}-\Phi_{0}\right), \\
s_{2} & =\sin 2\left(\frac{\pi}{2} \phi_{1}+\Omega_{1}-\Phi_{1}\right), \quad s_{4}=\sin 4\left(\frac{\pi}{2} \phi_{1}+\Omega_{0}-\Phi_{0}\right) .
\end{aligned}
$$

Similarly, matrix $\mathbf{G}_{H}^{*}$ can be decomposed as:

$$
\mathbf{G}_{H}^{*}=\underbrace{\left[\begin{array}{cc}
\Gamma & 0 \\
\operatorname{sym} & \Gamma
\end{array}\right]}_{\mathbf{G}_{H 0}^{*}}+\Lambda \rho_{1} \underbrace{\left[\begin{array}{cc}
c_{2}^{*} & s_{2}^{G_{H}^{*}} \\
\operatorname{sym} & -c_{2}^{G_{H}^{*}}
\end{array}\right]}_{\mathbf{G}_{H 1}^{*}}:=\mathbf{G}_{H 0}^{*}+\Lambda \rho_{1} \mathbf{G}_{H 1}^{*},
$$

with

$$
c_{2}^{G_{H}^{*}}=\cos 2\left(\Omega-\frac{\pi}{2} \phi_{1}+\Phi_{1}\right), \quad s_{2}^{G_{H}^{*}}=\sin 2\left(\Omega-\frac{\pi}{2} \phi_{1}+\Phi_{1}\right) .
$$


Since quasi-homogeneity holds, $\mathbf{G}_{B}^{*}=\mathbf{O}$ and $\mathbf{G}_{D}^{*}=\mathbf{G}_{A}^{*}$.

Let $h=t_{\mathrm{ply}} n_{0} n_{\mathrm{ref}}$, with reference to Eq. (4). Therefore, the following derivatives read:

$$
\begin{aligned}
\frac{\partial \mathbf{G}_{A}}{\partial n_{0}} & =\frac{h}{n_{0}} \mathbf{G}_{A}^{*}, \quad \frac{\partial \mathbf{G}_{A}}{\partial \rho_{0 K}}=h \Lambda_{0} \mathbf{G}_{A 1}^{*}, \quad \frac{\partial \mathbf{G}_{A}}{\partial \rho_{1}}=h \Lambda_{1} \mathbf{G}_{A 2}^{*}, \\
\frac{\partial \mathbf{G}_{A}}{\partial \phi_{1}} & =2 \pi h \Lambda_{0} \rho_{0 K}\left[\begin{array}{ccc}
-s_{4} & +s_{4} & c_{4} \\
& -s_{4} & -c_{4} \\
\operatorname{sym} & & +s_{4}
\end{array}\right]+\pi h \Lambda_{1} \rho_{1}\left[\begin{array}{ccc}
-4 s_{2} & 0 & 2 c_{2} \\
& +4 s_{2} & 2 c_{2} \\
\text { sym } & & 0
\end{array}\right] .
\end{aligned}
$$

Similarly, for matrix $\mathbf{G}_{D}$ and matrix $\mathbf{G}_{H}$ :

$$
\begin{aligned}
& \frac{\partial \mathbf{G}_{D}}{\partial n_{0}}=\frac{h^{2}}{4} \frac{\partial \mathbf{G}_{A}}{\partial n_{0}}, \quad \frac{\partial \mathbf{G}_{D}}{\partial \rho_{0 K}}=\frac{h^{2}}{12} \frac{\partial \mathbf{G}_{A}}{\partial \rho_{0 K}}, \quad \frac{\partial \mathbf{G}_{D}}{\partial \rho_{1}}=\frac{h^{2}}{12} \frac{\partial \mathbf{G}_{A}}{\partial \rho_{1}}, \quad \frac{\partial \mathbf{G}_{D}}{\partial \phi_{1}}=\frac{h^{2}}{12} \frac{\partial \mathbf{G}_{A}}{\partial \phi_{1}}, \\
& \frac{\partial \mathbf{G}_{H}}{\partial n_{0}}=\frac{h}{n_{0}} \mathbf{G}_{H}^{*}, \quad \frac{\partial \mathbf{G}_{H}}{\partial \rho_{0 K}}=\mathbf{O}, \quad \frac{\partial \mathbf{G}_{H}}{\partial \rho_{1}}=h \Lambda \mathbf{G}_{H 1}^{*}, \quad \frac{\partial \mathbf{H}}{\partial \phi_{1}}=-\pi h \Lambda \rho_{1}\left[\begin{array}{cc}
-s_{2}^{G_{H}^{*}} & c_{2}^{G_{H}^{*}} \\
\operatorname{sym} & s_{2}^{G_{H}^{*}}
\end{array}\right] .
\end{aligned}
$$

Finally, for the generic variable $\xi_{j}$, for an orthotropic quasi-homogeneous laminate,

$$
\frac{\partial \mathbf{G}}{\partial \xi_{j}}=\operatorname{diag}\left(\frac{\partial \mathbf{G}_{A}}{\partial \xi_{j}}, \frac{\partial \mathbf{G}_{D}}{\partial \xi_{j}}, \frac{\partial \mathbf{G}_{H}}{\partial \xi_{j}}\right) .
$$

\section{Analytic Expression of Geometric Stiffness Matrix Gradient and Buckling Factor Gradient}

The generalised eigenvalue problem for the LFEM can be stated as follows:

$$
\left(\mathbf{K}^{b}+\lambda \mathbf{K}_{\sigma}^{b}\right) \boldsymbol{\psi}_{b}=\mathbf{0}
$$

or, passing to work,

$$
\boldsymbol{\psi}_{b}^{T}\left(\mathbf{K}^{b}+\lambda \mathbf{K}_{\sigma}^{b}\right) \boldsymbol{\psi}_{b}=0
$$

where $\mathbf{K}^{b} \in \mathbb{M}_{s++}^{n_{I N}^{b} \times n_{\text {IN }}^{b}}$ is the (reduced) stiffness matrix of the LFEM, $\mathbf{K}_{\sigma}^{b} \in \mathbb{M}_{s+}^{n_{\mathrm{IN}}^{b} \times n_{\mathrm{IN}}^{b}}$ is the geometric stiffness matrix of the LFEM, $\lambda$ and $\boldsymbol{\psi}_{b}$ are the eigenvalue and eigenvector, respectively, non-trivial solution of problem (D.1). Note that $\mathbf{K}_{\sigma}^{b}$ is not, in general, positive-definite.

As usually done in classical buckling eigenvalue analyses, $\mathbf{K}_{\sigma}^{b}$ is calculated from the stress field solution of the (static) equilibrium boundary problem of the LFEM subject to the same BCs of the original eigenvalue buckling problem. However, in the framework of the considered GL modelling approach, the equilibrium boundary problem of the LFEM is of the Dirichlet's type: non-zero displacements are imposed at some DOFs, which can be collected in the set $I_{\mathrm{BC}}^{b}$ whose cardinality is $n_{\mathrm{BC}}^{b}$. On the other hand, the unknown DOFs are collected in the set $I_{\mathrm{IN}}^{\mathrm{b}}$ whose cardinality is $n_{\mathrm{IN}}^{b}$. Moreover, BCs depend on the displacement field solution of the GFEM equilibrium problem (13). Therefore, the key-point is to express properly the equilibrium displacement boundary problem (and the related derivatives) for the LFEM.

Definition D.1 Given a matrix $M \in \mathbb{M}^{\mathrm{m} \times \mathrm{n}}$ and the two sets of positive natural numbers $R \subset\{i \mid 1 \leq$ $i \leq \mathrm{m}\}$ and $C \subset\{j \mid 1 \leq j \leq \mathrm{n}\}$, the operator $\mathfrak{Z}(\boldsymbol{M}, R, C)$ returns a matrix obtained by annihilating the $i$-th row and the $j$-th column of $\boldsymbol{M}, \forall i \in R$ and $\forall j \in C$. Similarly, $\mathfrak{Z}(\boldsymbol{v}, R)$ denotes the vector obtained by annihilating the $i$-th component of vector $\boldsymbol{v} \in \mathbb{M}^{n \times 1}, \forall i \in R$. Operator $\mathfrak{Z}(\cdot)$ preserves the dimensions of its argument.

Since no external nodal forces are applied, the equilibrium equation of the LFEM reads

$$
\hat{\mathbf{K}}^{b} \hat{\mathbf{u}}_{0}^{b}=\hat{\mathbf{f}}_{0}^{b}
$$


where $\hat{\mathbf{K}}^{b} \in \mathbb{M}_{s}^{n_{\mathrm{DOF}}^{b} \times n_{\mathrm{DOF}}^{b}}$ is the (singular) stiffness matrix of the LFEM, $\hat{\mathbf{u}}_{0}^{b} \in \mathbb{M}^{n_{\mathrm{DOF}}^{b}} \times 1$ is the vector collecting the set of DOFs of the LFEM static analysis (both imposed displacements and unknown ones) and $\hat{\mathbf{f}}_{0}^{b} \in \mathbb{M}^{n_{\mathrm{DOF}}^{b}} \times 1$ is the vector of the unknown nodal forces (occurring at nodes where BCs on generalized displacements are applied). In the above expressions $n_{\mathrm{DOF}}^{b}=n_{\mathrm{BC}}^{b}+n_{\mathrm{IN}}^{b}$.

As discussed in Wu et al. (2007); Reddy (2005), problem (D.3) can be solved after a proper rearranging. In particular, if $\hat{u}_{s}$ (for some $s$ ) is assigned, one must set $\hat{K}_{s s}=1, \hat{K}_{i s}=\hat{K}_{s i}=0$ for $i \neq s$ and subtract to the right-hand side the $s$-th column of the (unmodified) stiffness matrix, multiplied by $\hat{u}_{s}$. After this operation, the new system can be reduced, as usually, and the unknown nodal displacements can be determined.

Remark D.1 Let $A \subset\{i \mid i=1, \ldots, n\}$ and $B \subset\{i \mid i=1, \ldots, n\}$ be two sets such that $A \cap B=\emptyset$ and $\sharp(A+B)=n$.

Therefore $\boldsymbol{u}=\mathfrak{Z}(\boldsymbol{u}, A) \oplus \mathfrak{Z}(\boldsymbol{u}, B), \forall \boldsymbol{u} \in \mathbb{M}^{n \times 1}$.

By applying Remark D.1 to $\hat{\mathbf{u}}_{0}^{b}$ and $\hat{\mathbf{f}}_{0}^{b}$ one obtains:

$$
\begin{gathered}
\hat{\mathbf{u}}_{0}^{b}=\mathfrak{Z}\left(\hat{\mathbf{u}}_{0}^{b}, I_{\mathrm{BC}}^{b}\right) \oplus \mathfrak{Z}\left(\hat{\mathbf{u}}_{0}^{b}, I_{\mathrm{IN}}^{b}\right):=\hat{\mathbf{u}}^{b}+\hat{\mathbf{u}}_{\mathrm{BC}}^{b} \\
\hat{\mathbf{f}}_{0}^{b}=\mathfrak{Z}\left(\hat{\mathbf{f}}_{0}^{b}, I_{\mathrm{BC}}^{b}\right) \oplus \mathfrak{Z}\left(\hat{\mathbf{f}}_{0}^{b}, I_{\mathrm{IN}}^{b}\right):=\hat{\mathbf{0}}+\hat{\mathbf{f}}_{\mathrm{BC}}^{b}
\end{gathered}
$$

where only vector $\hat{\mathbf{u}}_{\mathrm{BC}}^{b}$ is known. Therefore, problem (D.3) becomes

$$
\hat{\mathbf{K}}^{b} \hat{\mathbf{u}}^{b}+\hat{\mathbf{K}}^{b} \hat{\mathbf{u}}_{\mathrm{BC}}^{b}=\hat{\mathbf{0}}+\hat{\mathbf{f}}_{\mathrm{BC}}^{b}
$$

To solve for the unknown part of $\hat{\mathbf{u}}_{0}^{b}$, the operator $\mathfrak{R}$ of definition B.1 must be applied to Eq. (D.5): the resulting reduced system reads

$$
\mathbf{K}^{b} \mathbf{u}^{b}+\mathbf{K}_{\mathrm{BC}}^{b} \mathbf{u}_{\mathrm{BC}}^{b}=\mathbf{0}
$$

where $\mathbf{K}:=\mathfrak{R}\left(\hat{\mathbf{K}}^{b}, I_{\mathrm{BC}}^{b}, I_{\mathrm{BC}}^{b}\right), \mathbf{u}^{b}:=\mathfrak{R}\left(\hat{\mathbf{u}}_{0}^{b}, I_{\mathrm{BC}}^{b}\right), \mathbf{K}_{\mathrm{BC}}^{b}:=\mathfrak{R}\left(\hat{\mathbf{K}}, I_{\mathrm{BC}}^{b}, I_{\mathrm{IN}}^{b}\right), \mathbf{u}_{\mathrm{BC}}^{b}:=\mathfrak{R}\left(\hat{\mathbf{u}}_{0}^{b}, I_{\mathrm{IN}}^{b}\right)$ and $\mathfrak{R}\left(\hat{\mathbf{f}}_{\mathrm{BC}}^{b}, I_{\mathrm{BC}}^{b}\right)=\mathbf{0}$. Inasmuch as $\mathbf{u}_{\mathrm{BC}}^{b}$ depends on the GFEM solution, it is convenient to introduce the linear map

$$
\mathbf{P}: \mathbf{u} \mapsto \mathbf{u}_{\mathrm{BC}}^{b}, \quad \mathbf{P u}=\mathbf{u}_{\mathrm{BC}}^{b}, \quad \mathbf{P} \in \mathbb{M}^{n_{\mathrm{BC}}^{b} \times\left(n_{\mathrm{DOF}}-n_{\mathrm{BC}}\right)}
$$

whose aim is to determine the BCs to be imposed to the LFEM (in terms of nodal displacements), starting from the solution of the static analysis carried out on the GFEM. In particular, the number of nodes belonging to the boundary of the LFEM, where BCs are applied, is different (usually larger) than the number of nodes located on the same boundary where the known displacement field is extracted from the GFEM results. Furthermore, meshes may be completely dissimilar, as shown in Fig. 12 (boundary of LFEM in red, GFEM mesh in black). The construction of $\mathbf{P}$ can be done according to the steps listed in Algorithm 1, whose structure refers to the notation provided in Fig. 12. 


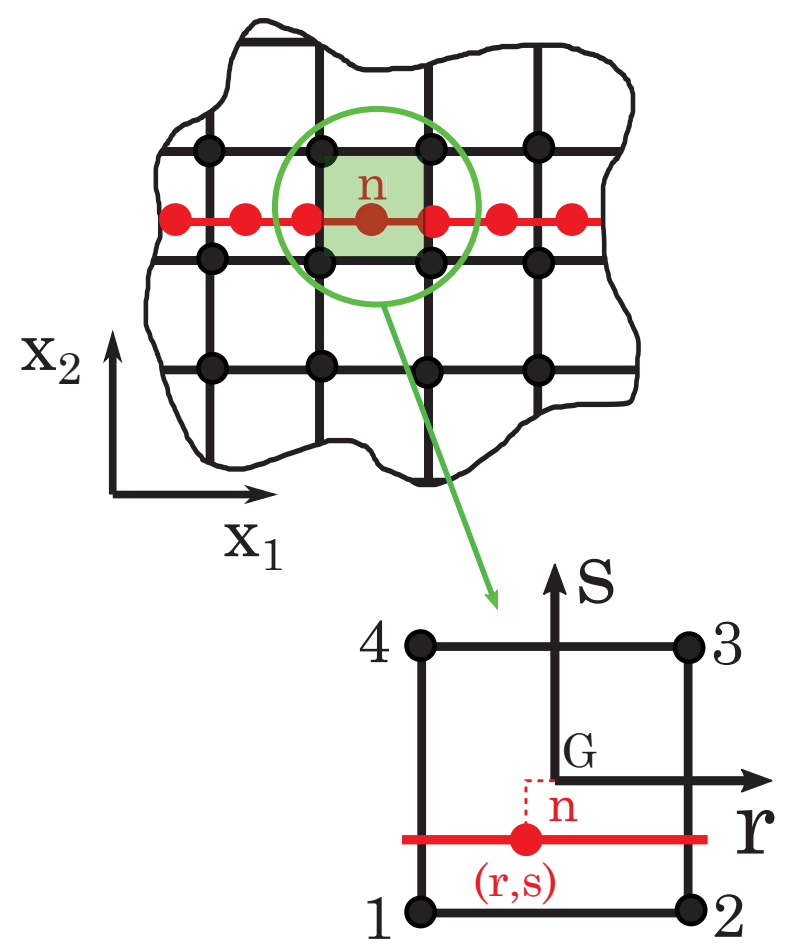

Figure 12: Differences between GFEM and LFEM meshes

\section{Algorithm 1 Generation of matrix P.}

1: Select a node (whose ID is $n$ in Fig. 12) located at the boundary of the LFEM where BCs are applied.

2: Get the node coordinates $\left(x_{n 1}, x_{n 2}\right)$ in the global frame.

3: Select the element of the GFEM containing the projection of node $n$ (green-shadowed in Fig. 12).

4: Evaluate the mapped coordinates $r$ and $s$ of node $n$ with respect to the principal frame of the element, placed at its centroid (frame $\{G, r, s\}$ in Fig. 12). The mapped coordinates are the results of the following problem:

$$
\mathbf{J}(r, s)\left(\begin{array}{l}
r \\
s
\end{array}\right)=\left(\begin{array}{l}
x_{n 1}-x_{G 1} \\
x_{n 2}-n_{G 2}
\end{array}\right)
$$

where $\mathbf{J} \in \mathbb{M}_{s++}^{2 \times 2}$ is the Jacobian matrix of the element, which depends on the unknowns $r$ and $s$. J maps the transformation of the square element into the (possibly) distorted element of the actual GFEM mesh.

5: Evaluate the matrix which maps the element nodal displacements (displacements of nodes 1, 2, 3 and 4) into the displacements at the point of coordinates $(r, s)$ (i.e., $\mathbf{u}_{\mathrm{BC}}^{b}(r, s)$ ). If $\mathbf{N}_{e} \in \mathbb{M}^{6 \times 24}$ is the matrix of the shape functions of the element (Barbero (2013); Reddy (2003)), the relation can be expressed as

$$
\mathbf{u}_{\mathrm{BC}}^{b}(r, s)=\mathbf{N}_{e}(r, s) \mathbf{u}_{e},
$$

where $\mathbf{u}_{e}$ is the vector of DOFs of the element $e$ belonging to the GFEM.

6: Properly assemble $\mathbf{N}_{e}(r, s)$ inside $\mathbf{P}$.

7: Repeat steps 1-6 for the remaining nodes belonging to the boundary of the LFEM where BCs on nodal displacements are applied. 
Taking into account for the above aspects, Eq. (D.6) reads:

$$
\mathbf{K}^{b} \mathbf{u}^{b}+\mathbf{K}_{\mathrm{BC}}^{b} \mathbf{P} \mathbf{u}=\mathbf{0}
$$

Consider, now, the augmented version of Eq. (D.2):

$$
\boldsymbol{\psi}_{b}^{\mathrm{T}}\left(\mathbf{K}^{\mathrm{b}}+\lambda \mathbf{K}_{\sigma}^{b}\right) \boldsymbol{\psi}_{b}+\boldsymbol{\mu}^{\mathrm{T}}\left(\mathbf{K}^{b} \mathbf{u}^{\mathrm{b}}+\mathbf{K}_{\mathrm{BC}}^{\mathrm{b}} \mathbf{P} \mathbf{u}\right)+\mathbf{w}^{\mathrm{T}}(\mathbf{K} \mathbf{u}-\mathbf{f})=0
$$

where $\boldsymbol{\mu} \neq \mathbf{0}$ and $\mathbf{w} \neq \mathbf{0}$ are the arbitrarily-defined adjoint vectors. By deriving Eq. (D.11) with respect to the generic design variable $\xi_{j}$, one obtains:

$$
\begin{aligned}
& \frac{\partial \lambda}{\partial \xi_{j}}=\frac{\lambda}{\boldsymbol{\psi}_{b}^{\mathrm{T}} \mathbf{K}^{\mathrm{b}} \boldsymbol{\psi}_{b}} {\left[\boldsymbol{\psi}_{b}^{\mathrm{T}}\left(\frac{\partial \mathbf{K}^{b}}{\partial \xi_{j}}+\lambda \frac{\partial \mathbf{K}_{\sigma}^{b}}{\partial \xi_{j}}\right) \boldsymbol{\psi}_{b}+\boldsymbol{\mu}^{\mathrm{T}}\left(\frac{\partial \mathbf{K}^{b}}{\partial \xi_{j}} \mathbf{u}^{\mathrm{b}}+\mathbf{K}^{\mathrm{b}} \frac{\partial \mathbf{u}^{\mathrm{b}}}{\partial \xi_{j}}+\cdots\right.\right.} \\
&\left.\left.\cdots+\frac{\partial \mathbf{K}_{\mathrm{BC}}^{\mathrm{b}}}{\partial \xi_{j}} \mathbf{P} \mathbf{u}+\mathbf{K}_{\mathrm{BC}}^{\mathrm{b}} \mathbf{P} \frac{\partial \mathbf{u}}{\partial \xi_{j}}\right)+\mathbf{w}^{\mathrm{T}}\left(\frac{\partial \mathbf{K}}{\partial \xi_{j}} \mathbf{u}+\mathbf{K} \frac{\partial \mathbf{u}}{\partial \xi_{j}}\right)\right]
\end{aligned}
$$

As discussed in Setoodeh et al. (2009), the geometric stiffness matrix of the generic shell element can be expressed as:

$$
\mathbf{K}_{\sigma e}^{b}=\sum_{i=1}^{8} r_{0 e i}^{b} \overline{\mathbf{K}}_{i}
$$

where $r_{0 e i}^{b}$ are the components of vector $\mathbf{r}$ of Eq. (2), resulting from the static analysis of Eq. (D.10) carried out on the LFEM, while $\overline{\mathbf{K}}_{i} \in \mathbb{M}_{s}^{24 \times 24}$ are matrices depending only on the geometry of the element. The algorithm for retrieving the expression of each matrix $\overline{\mathbf{K}}_{i}$ for a shell element with four nodes and six DOFs per node (like the SHELL181 ANSYS ${ }^{\circledR}$ shell element), whose kinematics is described in the framework of the FSDT, is presented. Of course, this algorithm must be executed off-line, i.e. before the optimisation process, once the element type has been selected.

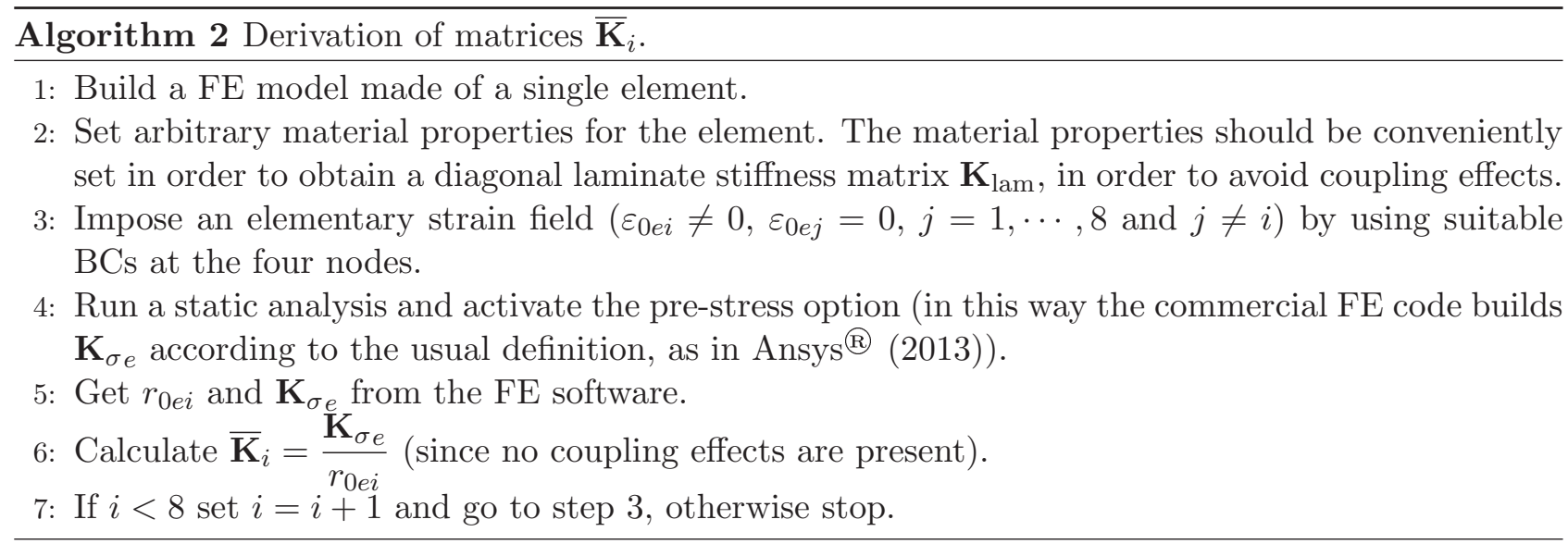

The expressions of $\overline{\mathbf{K}}_{i}$ for a square SHELL181 element of side $L$ are provided here below. Each matrix $\overline{\mathbf{K}}_{i}$ is a symmetric and sparse partitioned matrix, composed of symmetric blocks. Only non-null terms are provided in the following: 
$\overline{\mathbf{K}}_{1}=\frac{1}{8}\left[\begin{array}{cc}{\left[\begin{array}{cc}\hat{\mathbf{A}}_{1} & \hat{\mathbf{B}}_{1} \\ \hat{\mathbf{B}}_{1}^{\mathrm{T}} & \hat{\mathbf{C}}_{1}\end{array}\right]} & {\left[\begin{array}{cc}-\hat{\mathbf{A}}_{1} & -\hat{\mathbf{B}}_{1} \\ -\hat{\mathbf{B}}_{1}^{\mathrm{T}} & -\hat{\mathbf{C}}_{1}\end{array}\right]} \\ {\left[\begin{array}{cc}-\hat{\mathbf{A}}_{1} & -\hat{\mathbf{B}}_{1} \\ -\hat{\mathbf{B}}_{1}^{\mathrm{T}} & -\hat{\mathbf{C}}_{1}\end{array}\right]} & {\left[\begin{array}{cc}\hat{\mathbf{A}}_{1} & \hat{\mathbf{B}}_{1} \\ \hat{\mathbf{B}}_{1}^{\mathrm{T}} & \hat{\mathbf{C}}_{1}\end{array}\right]}\end{array}\right]$

with, $\hat{\mathbf{A}}_{1}=\hat{\mathbf{A}}_{1}^{\mathrm{T}}, \hat{\mathbf{C}}_{1}=\hat{\mathbf{C}}_{1}^{\mathrm{T}}$, and

$\hat{\mathrm{A}}_{1}^{(1,1)}=-3, \hat{\mathrm{A}}_{1}^{(1,2)}=-1, \hat{\mathrm{A}}_{1}^{(2,2)}=1, \hat{\mathrm{A}}_{1}^{(3,3)}=2$,

$\hat{\mathrm{B}}_{1}^{(1,1)}=1, \hat{\mathrm{B}}_{1}^{(1,2)}=1, \hat{\mathrm{B}}_{1}^{(2,1)}=-1, \hat{\mathrm{B}}_{1}^{(2,2)}=-1, \hat{\mathrm{B}}_{1}^{(3,3)}=-2$,

$\hat{\mathrm{C}}_{1}^{(1,1)}=-3, \hat{\mathrm{C}}_{1}^{(1,2)}=1, \hat{\mathrm{C}}_{1}^{(2,2)}=1, \hat{\mathrm{C}}_{1}^{(3,3)}=2$,

$\overline{\mathbf{K}}_{2}=\frac{1}{8}\left[\begin{array}{cc}{\left[\begin{array}{cc}\hat{\mathbf{A}}_{2} & \hat{\mathbf{B}}_{2} \\ \hat{\mathbf{B}}_{2}^{\mathrm{T}} & \hat{\mathbf{C}}_{2}\end{array}\right]} & {\left[\begin{array}{cc}-\hat{\mathbf{A}}_{2} & -\hat{\mathbf{B}}_{2} \\ -\hat{\mathbf{B}}_{2}^{\mathrm{T}} & -\hat{\mathbf{C}}_{2}\end{array}\right]} \\ {\left[\begin{array}{cc}-\hat{\mathbf{A}}_{2} & -\hat{\mathbf{B}}_{2} \\ -\hat{\mathbf{B}}_{2}^{\mathrm{T}} & -\hat{\mathbf{C}}_{2}\end{array}\right]} & {\left[\begin{array}{cc}\hat{\mathbf{A}}_{2} & \hat{\mathbf{B}}_{2} \\ \hat{\mathbf{B}}_{2}^{\mathrm{T}} & \hat{\mathbf{C}}_{2}\end{array}\right]}\end{array}\right]$,

with, $\hat{\mathbf{A}}_{2}=\hat{\mathbf{A}}_{2}^{\mathrm{T}}, \hat{\mathbf{C}}_{2}=\hat{\mathbf{C}}_{2}^{\mathrm{T}}$, and

$\hat{\mathrm{A}}_{2}^{(1,1)}=1, \hat{\mathrm{A}}_{2}^{(1,2)}=-1, \hat{\mathrm{A}}_{2}^{(2,2)}=-3, \hat{\mathrm{A}}_{2}^{(3,3)}=2$,

$\hat{\mathrm{B}}_{2}^{(1,1)}=1, \hat{\mathrm{B}}_{2}^{(1,2)}=1, \hat{\mathrm{B}}_{2}^{(2,1)}=-1, \hat{\mathrm{B}}_{2}^{(2,2)}=-1, \hat{\mathrm{B}}_{2}^{(3,3)}=2$,

$\hat{\mathrm{C}}_{2}^{(1,1)}=1, \hat{\mathrm{C}}_{2}^{(1,2)}=1, \hat{\mathrm{C}}_{2}^{(2,2)}=-3, \hat{\mathrm{C}}_{2}^{(3,3)}=2$,

$\overline{\mathbf{K}}_{3}=\frac{1}{2}\left[\begin{array}{cc}{\left[\begin{array}{cc}\hat{\mathbf{A}}_{3} & \mathbf{O} \\ \operatorname{sym} & \hat{\mathbf{C}}_{3}\end{array}\right]} & {\left[\begin{array}{cc}-\hat{\mathbf{A}}_{3} & \mathbf{O} \\ \mathrm{sym} & -\hat{\mathbf{C}}_{3}\end{array}\right]} \\ {\left[\begin{array}{cc}-\hat{\mathbf{A}}_{3} & \mathbf{O} \\ \mathrm{sym} & -\hat{\mathbf{C}}_{3}\end{array}\right]} & {\left[\begin{array}{cc}\hat{\mathbf{A}}_{3} & \mathbf{O} \\ \mathrm{sym} & \hat{\mathbf{C}}_{3}\end{array}\right]}\end{array}\right]$,

with, $\hat{\mathbf{A}}_{3}=\hat{\mathbf{A}}_{3}^{\mathrm{T}}, \hat{\mathbf{C}}_{3}=\hat{\mathbf{C}}_{3}^{\mathrm{T}}$, and

$\hat{\mathrm{A}}_{3}^{(1,2)}=-1, \hat{\mathrm{A}}_{3}^{(3,3)}=1$,

$\hat{\mathrm{C}}_{3}^{(1,2)}=-1, \hat{\mathrm{C}}_{3}^{(3,3)}=-1$,

$\overline{\mathbf{K}}_{4}=\overline{\mathbf{K}}_{5}=\overline{\mathbf{K}}_{6}=\mathbf{O}$,

$\overline{\mathbf{K}}_{7}=\frac{1}{72}\left[\begin{array}{cc}{\left[\begin{array}{cc}-24 \hat{\mathbf{K}}_{7}-12 \hat{\mathbf{A}}_{7}+4 \hat{\mathbf{C}}_{7} & 24 \hat{\mathbf{K}}_{7}+12 \hat{\mathbf{B}}_{7}+2 \hat{\mathbf{C}}_{7} \\ 24 \hat{\mathbf{K}}_{7}-12 \hat{\mathbf{B}}_{7}+2 \hat{\mathbf{C}}_{7} & -24 \hat{\mathbf{K}}_{7}+12 \hat{\mathbf{A}}_{7}+4 \hat{\mathbf{C}}_{7}\end{array}\right]} & {\left[\begin{array}{cc}12 \hat{\mathbf{K}}_{7}+6 \hat{\mathbf{B}}_{7}+\hat{\mathbf{C}}_{7} & -12 \hat{\mathbf{K}}_{7}-6 \hat{\mathbf{A}}_{7}+2 \hat{\mathbf{C}}_{7} \\ -12 \hat{\mathbf{K}}_{7}+6 \hat{\mathbf{A}}_{7}+2 \hat{\mathbf{C}}_{7} & 12 \hat{\mathbf{K}}_{7}-6 \hat{\mathbf{B}}_{7}+\hat{\mathbf{C}}_{7}\end{array}\right]} \\ {\left[\begin{array}{ccc}12 \hat{\mathbf{K}}_{7}-6 \hat{\mathbf{B}}_{7}+\hat{\mathbf{C}}_{7} & -12 \hat{\mathbf{K}}_{7}+6 \hat{\mathbf{A}}_{7}+2 \hat{\mathbf{C}}_{7} \\ -12 \hat{\mathbf{K}}_{7}-6 \hat{\mathbf{A}}_{7}+2 \hat{\mathbf{C}}_{7} & 12 \hat{\mathbf{K}}_{7}+6 \hat{\mathbf{B}}_{7}+\hat{\mathbf{C}}_{7}\end{array}\right]} & {\left[\begin{array}{cc}-24 \hat{\mathbf{K}}_{7}+12 \hat{\mathbf{A}}_{7}+4 \hat{\mathbf{C}}_{7} & 24 \hat{\mathbf{K}}_{7}-12 \hat{\mathbf{B}}_{7}+2 \hat{\mathbf{C}}_{7} \\ 24 \hat{\mathbf{K}}_{7}+12 \hat{\mathbf{B}}_{7}+2 \hat{\mathbf{C}}_{7} & -24 \hat{\mathbf{K}}_{7}-12 \hat{\mathbf{A}}_{7}+4 \hat{\mathbf{C}}_{7}\end{array}\right]}\end{array}\right]$,

with, $\hat{\mathbf{K}}_{7}=\hat{\mathbf{K}}_{7}^{\mathrm{T}}, \hat{\mathbf{A}}_{7}=\hat{\mathbf{A}}_{7}^{\mathrm{T}}, \hat{\mathbf{B}}_{7}=-\hat{\mathbf{B}}_{7}^{\mathrm{T}}, \hat{\mathbf{C}}_{7}=\hat{\mathbf{C}}_{7}^{\mathrm{T}}$, and

$\hat{\mathrm{K}}_{7}^{(1,3)}=1, \hat{\mathrm{A}}_{7}^{(1,5)}=L, \hat{\mathrm{B}}_{7}^{(1,5)}=-\hat{\mathrm{B}}_{7}^{(5,1)}=-L, \hat{\mathrm{C}}_{7}^{(4,6)}=L^{2}$, 


$$
\overline{\mathbf{K}}_{8}=\frac{1}{72}\left[\begin{array}{cc}
-12 \hat{\mathbf{K}}_{8}+6 \hat{\mathbf{A}}_{8}+2 \hat{\mathbf{C}}_{8}\left[\begin{array}{cc}
2 \mathbf{I} & \mathbf{I} \\
\mathbf{I} & 2 \mathbf{I}
\end{array}\right] & 12 \hat{\mathbf{K}}_{8}+6 \hat{\mathbf{B}}_{8}+\hat{\mathbf{C}}_{8}\left[\begin{array}{cc}
\mathbf{I} & 2 \mathbf{I} \\
2 \mathbf{I} & \mathbf{I}
\end{array}\right] \\
12 \hat{\mathbf{K}}_{8}-6 \hat{\mathbf{B}}_{8}+\hat{\mathbf{C}}_{8}\left[\begin{array}{cc}
\mathbf{I} & 2 \mathbf{I} \\
2 \mathbf{I} & \mathbf{I}
\end{array}\right] & -12 \hat{\mathbf{K}}_{8}-6 \hat{\mathbf{A}}_{8}+2 \hat{\mathbf{C}}_{8}\left[\begin{array}{cc}
2 \mathbf{I} & \mathbf{I} \\
\mathbf{I} & 2 \mathbf{I}
\end{array}\right]
\end{array}\right],
$$

with, $\hat{\mathbf{K}}_{8}=\hat{\mathbf{K}}_{8}^{\mathrm{T}}, \hat{\mathbf{A}}_{8}=\hat{\mathbf{A}}_{8}^{\mathrm{T}}, \hat{\mathbf{B}}_{8}=-\hat{\mathbf{B}}_{8}^{\mathrm{T}}, \hat{\mathbf{C}}_{8}=\hat{\mathbf{C}}_{8}^{\mathrm{T}}$, and

$$
\hat{\mathrm{K}}_{8}^{(2,3)}=1, \hat{\mathrm{A}}_{8}^{(2,4)}=L, \hat{\mathrm{B}}_{8}^{(2,4)}=-\hat{\mathrm{B}}_{8}^{(4,2)}=L, \hat{\mathrm{C}}_{8}^{(5,6)}=L^{2},
$$

The expressions of matrices $\overline{\mathbf{K}}_{i}$ reported above are supposed independent from the aspect ratio of the element. Of course, this assumption is justified if and only if the mesh of the FE model is structured and regular as much as possible (i.e. composed by pseudo-square elements). Accordingly, the singular form of the geometric stiffness matrix reads:

$$
\hat{\mathbf{K}}_{\sigma}^{b}=\sum_{e=1}^{N_{e}^{b}} \hat{\mathbf{L}}_{e} \sum_{i=1}^{\mathrm{T}} \sum_{0 e i}^{b} \overline{\mathbf{K}}_{i} \hat{\mathbf{L}}_{e}^{b}=\sum_{e=1}^{N_{e}^{b}} \hat{\mathbf{L}}_{e}^{b \mathrm{~T}} \sum_{i=1}^{8}\left(\mathbf{K}_{e}^{l a m} \mathbf{B}_{e} \mathbf{u}_{e 0}^{b}\right)_{i} \overline{\mathbf{K}}_{i} \hat{\mathbf{L}}_{e}^{b}=\sum_{e=1}^{N_{e}^{b}} \hat{\mathbf{L}}_{e}^{b \mathrm{~T}} \sum_{i=1}^{8}\left(\mathbf{K}_{e}^{l a m} \mathbf{B}_{e} \hat{\mathbf{L}}_{e}^{b} \hat{\mathbf{u}}_{0}^{b}\right)_{i} \overline{\mathbf{K}}_{i} \hat{\mathbf{L}}_{e}^{b},
$$

and the non-singular counterpart can be obtained as

$$
\mathbf{K}_{\sigma}^{b}:=\Re\left(\hat{\mathbf{K}}_{\sigma}^{b}, I_{\mathrm{BC}}^{b}, I_{\mathrm{BC}}^{\mathrm{b}}\right) .
$$

Consider the following quantity:

$$
\begin{aligned}
\hat{\boldsymbol{\psi}}_{b}^{\mathrm{T}} \frac{\partial \hat{\mathbf{K}}_{\sigma}^{\mathrm{b}}}{\partial \xi_{j}} \hat{\boldsymbol{\psi}}_{b} & =\hat{\boldsymbol{\psi}}_{b}^{\mathrm{T}}\left(\sum_{e=1}^{N_{e}^{\mathrm{b}}} \hat{\mathbf{L}}_{e}^{\mathrm{T}}\left(\sum_{i=1}^{8} \frac{\partial}{\partial \xi_{j}}\left(\mathbf{K}_{e}^{l a m} \mathbf{B}_{e} \mathbf{u}_{e 0}^{\mathrm{b}}\right)_{i} \overline{\mathbf{K}}_{i}\right) \hat{\mathbf{L}}_{e}^{b}\right) \hat{\boldsymbol{\psi}}_{b} \\
& =\sum_{e=1}^{N_{e}^{\mathrm{b}}} \hat{\boldsymbol{\psi}}_{b}^{\mathrm{T}} \hat{\mathbf{L}}_{e}^{\mathrm{T}}\left(\sum_{i=1}^{8} \frac{\partial}{\partial \xi_{j}}\left(\mathbf{K}_{e}^{l a m} \mathbf{B}_{e} \mathbf{u}_{e 0}^{\mathrm{b}}\right)_{i} \overline{\mathbf{K}}_{i}\right) \hat{\mathbf{L}}_{e}^{\mathrm{b}} \hat{\boldsymbol{\psi}}_{b} \\
& =\sum_{e=1}^{N_{e}^{b}} \boldsymbol{\psi}_{e b}^{\mathrm{T}}\left(\sum_{i=1}^{8} \frac{\partial}{\partial \xi_{j}}\left(\mathbf{K}_{e}^{\text {lam }} \mathbf{B}_{e} \mathbf{u}_{e 0}^{\mathrm{b}}\right)_{i} \overline{\mathbf{K}}_{i}\right) \boldsymbol{\psi}_{e b} \\
& =\sum_{e=1}^{N_{e}^{b}} \sum_{i=1}^{8} \frac{\partial}{\partial \xi_{j}}\left(\mathbf{K}_{e}^{l a m} \mathbf{B}_{e} \mathbf{u}_{e 0}^{b}\right)_{i} \boldsymbol{\psi}_{e b}^{\mathrm{T}} \overline{\mathbf{K}}_{i} \boldsymbol{\psi}_{e b} \\
& =\sum_{e=1}^{N_{e}^{b}} \mathbf{s}_{e b}^{\mathrm{T}} \frac{\partial}{\partial \xi_{j}}\left(\mathbf{K}_{e}^{l a m} \mathbf{B}_{e} \mathbf{u}_{e 0}^{b}\right) \\
& =\sum_{e=1}^{N_{e}^{b}} \mathbf{s}_{e b}^{\mathrm{T}} \frac{\partial \mathbf{K}_{e}^{l a m}}{\partial \xi_{j}} \mathbf{B}_{e} \hat{\mathbf{L}}_{e}^{b} \hat{\mathbf{u}}_{0}^{b}+\underbrace{\sum_{e=1}^{N_{e}^{b}} \mathbf{s}_{e b}^{\mathrm{T}} \mathbf{K}_{e}^{l a m} \mathbf{B}_{e} \hat{\mathbf{L}}_{e}^{b}}_{\hat{\mathbf{a}}^{\mathrm{T}}} \frac{\partial \hat{\mathbf{u}}_{0}^{b}}{\partial \xi_{j}} \\
& :=\hat{\mathbf{a}}^{\mathrm{T}} \hat{\mathbf{u}}_{0}^{b}+\hat{\mathbf{b}}^{\mathrm{T}} \frac{\partial \hat{\mathbf{u}}_{0}^{b}}{\partial \xi_{j}},
\end{aligned}
$$

with $\mathbf{s}_{e b}:=\left\{\boldsymbol{\psi}_{e b}^{\mathrm{T}} \overline{\mathbf{K}}_{i} \boldsymbol{\psi}_{e b} \mid i=1, \ldots, 8\right\}$

Remark D.2 Consider the scalar product $\boldsymbol{v}^{\mathrm{T}} \boldsymbol{u}$ of two vectors $\boldsymbol{u}, \boldsymbol{v} \in \mathbb{M}^{n \times 1}$. If $\boldsymbol{u}, A$ and $B$ satisfies conditions of Remark D.1, then: $\boldsymbol{v}^{\mathrm{T}} \boldsymbol{u}=\boldsymbol{v}^{\mathrm{T}} \mathfrak{Z}(\boldsymbol{u}, A) \oplus \boldsymbol{v}^{\mathrm{T}} \mathfrak{Z}(\boldsymbol{u}, B)=\mathfrak{R}(\boldsymbol{v}, A)^{\mathrm{T}} \mathfrak{R}(\boldsymbol{u}, A) \oplus \mathfrak{R}(\boldsymbol{v}, B)^{\mathrm{T}} \mathfrak{R}(\boldsymbol{u}, B)$. 
By applying Remarks D.1 and D.2 to both $\hat{\boldsymbol{\psi}}^{b}$ and $\hat{\mathbf{u}}_{0}^{b}$ of Eq. (D.22), considering that $\mathfrak{R}\left(\hat{\boldsymbol{\psi}}^{b}, I_{\mathrm{IN}}^{b}\right)=\mathbf{0}$, one obtains:

$$
\begin{aligned}
\boldsymbol{\psi}_{b}^{\mathrm{T}} \frac{\partial \mathbf{K}_{\sigma}^{b}}{\partial \xi_{j}} \boldsymbol{\psi}_{b} & =\mathfrak{R}\left(\hat{\mathbf{a}}, I_{\mathrm{IN}}^{b}\right)^{\mathrm{T}} \mathfrak{R}\left(\hat{\mathbf{u}}_{0}^{b}, I_{\mathrm{IN}}^{b}\right)+\mathfrak{R}\left(\hat{\mathbf{a}}, I_{\mathrm{BC}}^{b}\right)^{\mathrm{T}} \mathfrak{R}\left(\hat{\mathbf{u}}_{0}^{b}, I_{\mathrm{BC}}^{b}\right)+\mathfrak{R}\left(\hat{\mathbf{b}}, I_{\mathrm{IN}}^{b}\right)^{\mathrm{T}} \mathfrak{R}\left(\frac{\partial \hat{\mathbf{u}}_{0}^{b}}{\partial \xi_{j}}, I_{\mathrm{IN}}^{b}\right)+\cdots \\
& \cdots+\mathfrak{R}\left(\hat{\mathbf{b}}, I_{\mathrm{BC}}^{b}\right)^{\mathrm{T}} \mathfrak{R}\left(\frac{\partial \hat{\mathbf{u}}_{0}^{b}}{\partial \xi_{j}}, I_{\mathrm{BC}}^{b}\right)= \\
= & \Re\left(\hat{\mathbf{a}}, I_{\mathrm{IN}}^{b}\right)^{\mathrm{T}} \mathbf{u}_{\mathrm{BC}}^{b}+\mathfrak{R}\left(\hat{\mathbf{a}}, I_{\mathrm{BC}}^{b}\right)^{\mathrm{T}} \mathbf{u}^{b}+\mathfrak{R}\left(\hat{\mathbf{b}}, I_{\mathrm{IN}}^{b}\right)^{\mathrm{T}} \frac{\partial \mathbf{u}_{\mathrm{BC}}^{b}}{\partial \xi_{j}}+\mathfrak{R}\left(\hat{\mathbf{b}}, I_{\mathrm{BC}}^{b}\right)^{\mathrm{T}} \frac{\partial \mathbf{u}^{b}}{\partial \xi_{j}}= \\
= & \Re\left(\hat{\mathbf{a}}, I_{\mathrm{IN}}^{b}\right)^{\mathrm{T}} \mathbf{P} \mathbf{u}+\mathfrak{R}\left(\hat{\mathbf{a}}, I_{\mathrm{BC}}^{b}\right)^{\mathrm{T}} \mathbf{u}^{b}+\mathfrak{R}\left(\hat{\mathbf{b}}, I_{\mathrm{IN}}^{b}\right)^{\mathrm{T}} \mathbf{P} \frac{\partial \mathbf{u}}{\partial \xi_{j}}+\mathfrak{R}\left(\hat{\mathbf{b}}, I_{\mathrm{BC}}^{b}\right)^{\mathrm{T}} \frac{\partial \mathbf{u}^{b}}{\partial \xi_{j}},
\end{aligned}
$$

By injecting Eq. (D.23) into Eq. (D.11), and by choosing $\boldsymbol{\mu}$ and $\mathbf{w}$ such that the terms multiplying $\partial \mathbf{u}^{\mathrm{b}} / \partial \xi_{j}$ and $\partial \mathbf{u} / \partial \xi_{j}$ vanish, one finally obtains:

$$
\left\{\begin{array}{l}
\frac{\partial \lambda}{\partial \xi_{j}}=\frac{\lambda}{\boldsymbol{\psi}_{\mathrm{b}}^{\mathrm{T}} \mathbf{K}^{\mathrm{b}} \boldsymbol{\psi}_{b}}\left[\boldsymbol{\psi}_{\mathrm{b}}^{\mathrm{T}} \frac{\partial \mathbf{K}^{b}}{\partial \xi_{j}} \boldsymbol{\psi}_{b}+\lambda\left(\mathfrak{R}\left(\hat{\mathbf{a}}, I_{\mathrm{IN}}^{\mathrm{b}}\right)^{\mathrm{T}} \mathbf{P} \mathbf{u}+\mathfrak{R}\left(\hat{\mathbf{a}}, I_{\mathrm{BC}}^{\mathrm{b}}\right)^{\mathrm{T}} \mathbf{u}^{\mathrm{b}}\right)+\boldsymbol{\mu}^{\mathrm{T}}\left(\frac{\partial \mathbf{K}^{b}}{\partial \xi_{j}} \mathbf{u}^{\mathrm{b}}+\frac{\partial \mathbf{K}_{\mathrm{BC}}^{b}}{\partial \xi_{j}} \mathbf{P} \mathbf{u}\right)+\mathbf{w}^{\mathrm{T}} \frac{\partial \mathbf{K}}{\partial \xi_{j}} \mathbf{u}\right] \\
j=1, \ldots, n_{\mathrm{vars}} \\
\mathbf{K}^{b} \boldsymbol{\mu}=-\lambda \Re\left(\hat{\mathbf{b}}, I_{\mathrm{BC}}^{\mathrm{b}}\right) \\
\mathbf{K} \mathbf{w}=-\mathbf{P}^{\mathrm{T}}\left[\lambda \Re\left(\hat{\mathbf{b}}, I_{\mathrm{IN}}^{b}\right)+\mathbf{K}_{\mathrm{BC}}^{\mathrm{T}} \boldsymbol{\mu}\right] .
\end{array}\right.
$$

Eq. (D.24) represents the gradient of the buckling factor of the LFEM subject to non-null imposed BCs, which are related to the displacement field solution of static analysis performed on the GFEM. The last term of the first formula in Eq. (D.24) is the coupling effect between GFEM and LFEM and is non-zero $\forall j=1, \cdots, n_{\text {vars }}$. Conversely, the other terms are non-zero if and only if the design variable $\xi_{j}$ is defined in the LFEM domain.

\section{References}

Albazzan MA, Harik R, Tatting BF, Gürdal Z (2019) Efficient design optimization of nonconventional laminated composites using lamination parameters: A state of the art. Composite Structures 209:362-374, DOI 10.1016/j.compstruct.2018.10.095

Ansys ${ }^{\circledR}$ (2013) ANSYS ${ }^{\circledR}$ Mechanical APDL Basic Analysis Guide. Release 15.0. ANSYS Inc, Southpointe, 257 Technology Drive: Canonsburg, PA 15317

Barbero EJ (2013) Finite Element Analysis of Composite Materials Using ANSYS ${ }^{\circledR}$. Taylor \& Francis Inc

Bendsøe MP, Sigmund O (2004) Topology Optimization. Springer Berlin Heidelberg, DOI 10.1007/ 978-3-662-05086-6

Bian X, Fang Z (2017) Large-scale buckling-constrained topology optimization based on assemblyfree finite element analysis. Advances in Mechanical Engineering 9(9):168781401771542, DOI $10.1177 / 1687814017715422$

Bisagni C, Vescovini R (2015) A fast procedure for the design of composite stiffened panels. The Aeronautical Journal 119(1212):185-201, DOI 10.1017/s0001924000010332

Calafiore G, El Ghaoui L (2014) Optimization Models. Cambridge University Press 
Catapano A (2013) Stiffness and strength optimisation of the anisotropy distribution for laminated structures. PhD thesis, Université Pierre et Marie Curie - Paris VI, URL https://tel. archives-ouvertes.fr/tel-00952372/document, english

Catapano A, Montemurro M (2014a) A multi-scale approach for the optimum design of sandwich plates with honeycomb core. part i: homogenisation of core properties. Composite Structures 118:664-676, DOI 10.1016/j.compstruct.2014.07.057

Catapano A, Montemurro M (2014b) A multi-scale approach for the optimum design of sandwich plates with honeycomb core. part II: the optimisation strategy. Composite Structures 118:677-690, DOI 10.1016/j.compstruct.2014.07.058

Catapano A, Montemurro M (2018) On the correlation between stiffness and strength properties of anisotropic laminates. Mechanics of Advanced Materials and Structures 26(8):651-660, DOI 10.1080/15376494.2017.1410906

Catapano A, Desmorat B, Vannucci P (2012) Invariant formulation of phenomenological failure criteria for orthotropic sheets and optimisation of their strength. Mathematical Methods in the Applied Sciences 35(15):1842-1858, DOI 10.1002/mma.2530

Catapano A, Desmorat B, Vannucci P (2014) Stiffness and strength optimization of the anisotropy distribution for laminated structures. Journal of Optimization Theory and Applications 167(1):118146, DOI 10.1007/s10957-014-0693-5

Ciampa PD, Nagel B, Tooren M (2010) Global local structural optimization of transportation aircraft wings. In: 51st AIAA/ASME/ASCE/AHS/ASC Structures, Structural Dynamics, and Materials Conference, American Institute of Aeronautics and Astronautics, DOI 10.2514/6.2010-3098

Costa G, Montemurro M, Pailhès J (2017) A 2d topology optimisation algorithm in NURBS framework with geometric constraints. International Journal of Mechanics and Materials in Design 14(4):669696, DOI 10.1007/s10999-017-9396-z

Costa G, Montemurro M, Pailhès J (2019a) NURBS hyper-surfaces for 3d topology optimization problems. Mechanics of Advanced Materials and Structures pp 1-20, DOI 10.1080/15376494.2019. 1582826

Costa G, Montemurro M, Pailhès J, Perry N (2019b) Maximum length scale requirement in a topology optimisation method based on NURBS hyper-surfaces. CIRP Annals 68(1):153-156, DOI 10.1016/j.cirp.2019.04.048

Ferrari F, Sigmund O (2019) Revisiting topology optimization with buckling constraints. Structural and Multidisciplinary Optimization 59(5):1401-1415, DOI 10.1007/s00158-019-02253-3

Haykin S (1998) Neural Networks: A Comprehensive Foundation. Prentice Hall

Herencia JE, Weaver PM, Friswell MI (2008) Initial sizing optimisation of anisotropic composite panels with t-shaped stiffeners. Thin-Walled Structures 46(4):399-412, DOI 10.1016/j.tws.2007.09.003

IJsselmuiden ST, Abdalla MM, Seresta O, Gürdal Z (2009) Multi-step blended stacking sequence design of panel assemblies with buckling constraints. Composites Part B: Engineering 40(4):329-336, DOI 10.1016/j.compositesb.2008.12.002

Ijsselmuiden ST, Abdalla MM, Gürdal Z (2010) Optimization of variable-stiffness panels for maximum buckling load using lamination parameters. AIAA Journal 48(1):134-143, DOI 10.2514/1.42490

Irisarri FX, Laurin F, Leroy FH, Maire JF (2011) Computational strategy for multiobjective optimization of composite stiffened panels. Composite Structures 93(3):1158-1167, DOI 10.1016/j. compstruct.2010.10.005 
Izzi MI, Montemurro M, Catapano A, Pailhès J (2020) A multi-scale two-level optimisation strategy integrating a global/local modelling approach for composite structures. Composite Structures DOI 10.1016/j.compstruct.2020.111908

Jones RM (2018) Mechanics of Composite Materials. CRC Press, DOI 10.1201/9781498711067

Kristinsdottir BP, Zabinsky ZB, Tuttle ME, Neogi S (2001) Optimal design of large composite panels with varying loads. Composite Structures 51(1):93-102, DOI 10.1016/s0263-8223(00)00128-8

Lehoucq RB, Sorensen DC, Yang C (1998) ARPACK Users Guide. Society for Industrial and Applied Mathematics, DOI 10.1137/1.9780898719628

Liu B, Haftka R, Akgün M (2000) Two-level composite wing structural optimization using response surfaces. Structural and Multidisciplinary Optimization 20(2):87-96, DOI 10.1007/s001580050140

Liu D, Toroporov VV, Querin OM, Barton DC (2011) Bilevel optimization of blended composite wing panels. Journal of Aircraft 48(1):107-118, DOI 10.2514/1.c000261

Liu Q, Jrad M, Mulani SB, Kapania RK (2016) Global/local optimization of aircraft wing using parallel processing. AIAA Journal 54(11):3338-3348, DOI 10.2514/1.j054499

Liu S, Hou Y, Sun X, Zhang Y (2012) A two-step optimization scheme for maximum stiffness design of laminated plates based on lamination parameters. Composite Structures 94(12):3529-3537, DOI 10.1016/j.compstruct.2012.06.014

Mao KM, Sun CT (1991) A refined global-local finite element analysis method. International Journal for Numerical Methods in Engineering 32(1):29-43, DOI 10.1002/nme.1620320103

Montemurro M (2015a) An extension of the polar method to the First-order Shear Deformation Theory of laminates. Composite Structures 127:328-339, DOI 10.1016/j.compstruct.2015.03.025

Montemurro M (2015b) Corrigendum to "An extension of the polar method to the First-order Shear Deformation Theory of laminates" . Composite Structures 131:1143-1144, DOI 10.1016/j. compstruct.2015.06.002

Montemurro M (2015c) The polar analysis of the third-order shear deformation theory of laminates. Composite Structures 131:775-789, DOI 10.1016/j.compstruct.2015.06.016

Montemurro M, Catapano A (2016) A new paradigm for the optimum design of variable angle tow laminates. In: Variational Analysis and Aerospace Engineering, Springer International Publishing, pp 375-400, DOI 10.1007/978-3-319-45680-5\_14

Montemurro M, Catapano A (2017) On the effective integration of manufacturability constraints within the multi-scale methodology for designing variable angle-tow laminates. Composite Structures 161:145-159, DOI 10.1016/j.compstruct.2016.11.018

Montemurro M, Catapano A (2019) A general b-spline surfaces theoretical framework for optimisation of variable angle-tow laminates. Composite Structures 209:561-578, DOI 10.1016/j.compstruct. 2018.10.094

Montemurro M, Vincenti A, Vannucci P (2012a) A two-level procedure for the global optimum design of composite modular structures - application to the design of an aircraft wing. Journal of Optimization Theory and Applications 155(1):1-23, DOI 10.1007/s10957-012-0067-9

Montemurro M, Vincenti A, Vannucci P (2012b) A two-level procedure for the global optimum design of composite modular structures - application to the design of an aircraft wing. Journal of Optimization Theory and Applications 155(1):24-53, DOI 10.1007/s10957-012-0070-1 
Montemurro M, Vincenti A, Koutsawa Y, Vannucci P (2013) A two-level procedure for the global optimization of the damping behavior of composite laminated plates with elastomer patches. Journal of Vibration and Control 21(9):1778-1800, DOI 10.1177/1077546313503358

Montemurro M, Catapano A, Doroszewski D (2016) A multi-scale approach for the simultaneous shape and material optimisation of sandwich panels with cellular core. Composites Part B: Engineering 91:458-472, DOI 10.1016/j.compositesb.2016.01.030

Montemurro M, Pagani A, Fiordilino GA, Pailhès J, Carrera E (2018) A general multi-scale two-level optimisation strategy for designing composite stiffened panels. Composite Structures 201:968-979, DOI 10.1016/j.compstruct.2018.06.119

Montemurro M, Izzi MI, El-Yagoubi J, Fanteria D (2019) Least-weight composite plates with unconventional stacking sequences: Design, analysis and experiments. Journal of Composite Materials 53(16):2209-2227, DOI 10.1177/0021998318824783

Munk DJ, Vio GA, Steven GP (2016) A simple alternative formulation for structural optimisation with dynamic and buckling objectives. Structural and Multidisciplinary Optimization 55(3):969986, DOI 10.1007/s00158-016-1544-9

Neves MM, Rodrigues H, Guedes JM (1995) Generalized topology design of structures with a buckling load criterion. Structural Optimization 10(2):71-78, DOI 10.1007/bf01743533

Nielsen F, Sun K (2016) Guaranteed bounds on information-theoretic measures of univariate mixtures using piecewise log-sum-exp inequalities. Entropy 18(12):442, DOI 10.3390/e18120442

Panettieri E, Montemurro M, Catapano A (2019) Blending constraints for composite laminates in polar parameters space. Composites Part B: Engineering 168:448-457, DOI 10.1016/j.compositesb. 2019.03.040

Ramirez C, Sanchez R, Kreinovich V, Argaez M (2014) $\sqrt{x^{2}+\mu}$ is the most computationally efficient smooth approximation to $|x|$ : A proof. Journal of Uncertain Systems 8, URL https: //core.ac.uk/download/pdf/46739739.pdf

Reddy JN (2003) Mechanics of Laminated Composite Plates and Shells: Theory and Analysis, Second Edition. CRC Press

Reddy JN (2005) An Introduction to the Finite Element Method (McGraw-Hill Mechanical Engineering). McGraw-Hill Education

Rodrigues HC, Guedes JM, Bendsøe MP (1995) Necessary conditions for optimal design of structures with a nonsmooth eigenvalue based criterion. Structural Optimization 9(1):52-56, DOI $10.1007 / \mathrm{bf} 01742645$

Rudin W (1976) Principles of Mathematical Analysis. McGraw-Hill Education - Europe

Setoodeh S, Abdalla MM, IJsselmuiden ST, Gürdal Z (2009) Design of variable-stiffness composite panels for maximum buckling load. Composite Structures 87(1):109-117, DOI 10.1016/j.compstruct. 2008.01.008

Sun C, Mao K (1988) A global-local finite element method suitable for parallel computations. Computers \& Structures 29(2):309-315, DOI 10.1016/0045-7949(88)90264-7

The MathWork Inc (2011) Optimization toolbox user's guide

Thomsen CR, Wang F, Sigmund O (2018) Buckling strength topology optimization of 2d periodic materials based on linearized bifurcation analysis. Computer Methods in Applied Mechanics and Engineering 339:115-136, DOI 10.1016/j.cma.2018.04.031 
Townsend S, Kim HA (2019) A level set topology optimization method for the buckling of shell structures. Structural and Multidisciplinary Optimization DOI 10.1007/s00158-019-02374-9

Tsai S, Hahn T (1980) Introduction to composite materials. Technomic

Tsai S, Pagano NJ (1968) Invariant properties of composite materials. Tech. rep., Air force materials lab Wright-Patterson AFB Ohio

Vankan WJ, Maas R, Grihon S (2014) Efficient optimisation of large aircraft fuselage structures. The Aeronautical Journal 118(1199):31-52, DOI 10.1017/s0001924000008915

Vannucci P (2005) Plane anisotropy by the polar method. Meccanica 40(4-6):437-454, DOI $10.1007 / \mathrm{s} 11012-005-2132-\mathrm{z}$

Vannucci P (2012) A note on the elastic and geometric bounds for composite laminates. Journal of Elasticity 112(2):199-215, DOI 10.1007/s10659-012-9406-1

Vannucci P (2017) Anisotropic Elasticity. Springer-Verlag GmbH

Venkataraman S, Haftka R (2004) Structural optimization complexity: what has moore's law done for us? Structural and Multidisciplinary Optimization 28(6):375-387, DOI 10.1007/s00158-004-0415-y

Verchery G (1982) Les invariants des tenseurs d'ordre 4 du type de l'élasticité. In: Mechanical Behavior of Anisotropic Solids / Comportment Méchanique des Solides Anisotropes, Springer Netherlands, pp 93-104, DOI 10.1007/978-94-009-6827-1\_7

Whitcomb J (1991) Iterative global/local finite element analysis. Computers \& Structures 40(4):10271031, DOI 10.1016/0045-7949(91)90334-i

Wu B, Xu Z, Li Z (2007) A note on imposing displacement boundary conditions in finite element analysis. Communications in Numerical Methods in Engineering 24(9):777-784, DOI 10.1002/cnm.989

Ye HL, Wang WW, Chen N, Sui YK (2015) Plate/shell topological optimization subjected to linear buckling constraints by adopting composite exponential filtering function. Acta Mechanica Sinica 32(4):649-658, DOI 10.1007/s10409-015-0531-5

Zhang B, Dai R, Ma W, Wu H, Jiang L, Yan C, Zhang Y (2019) Analysis and design of carbon fibre clamping apparatus for replacement of insulator strings in ultra-high voltage transmission line. The Journal of Engineering 2019(16):2212-2215, DOI 10.1049/joe.2018.8907 